\title{
Early Detection of Parkinson's Disease Using Image Processing and Artificial Neural Network
}

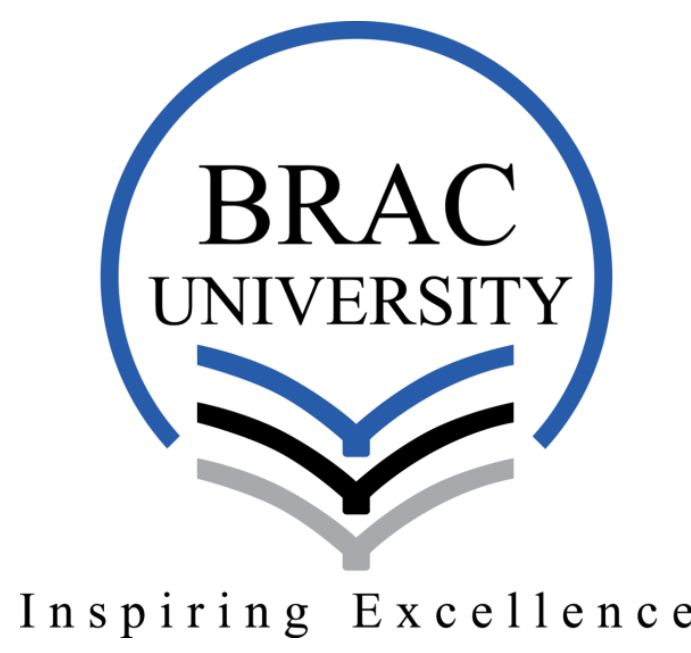

Mosarrat Rumman

14101080

Abu Nayeem Tasneem

14101121

Sadia Farzana

14101128

Department of Computer Science and Engineering

Supervisor:

Dr. Md. Ashraful Alam

Assistant Professor

Department of Computer Science and Engineering 


\section{DECLARATION}

We, hereby declare that this thesis is based on results we have found ourselves. Materials of work from researchers conducted by others are mentioned in references.

Mosarrat Rumman isaba2894@gmail.com

Abu Nayeem Tasneem tasneemabir@gmail.com

$\underline{\text { Sadia Farzana }}$

sadia.farzana013@gmail.com 


\begin{abstract}
Early detection of Parkinson's Disease (PD) is very crucial for effective management and treatment of the disease. Dopaminergic images such as Single Photon Emission Tomography (SPECT) using ${ }^{123}$ I-Ioflupane can substantially detect Parkinson's Disease at an early stage. However, till today, these images are mostly interpreted by humans which can manifest interobserver variability and inconsistency. To improve the imaging diagnosis of PD, we propose a model in this paper, for early detection of Parkinson's disease using Image Processing and Artificial Neural Network (ANN). The model used 200 SPECT images, 100 of healthy normal and 100 of PD, obtained from Parkinson's Progression Marker's Initiative (PPMI) database and processed them to find the area of Caudate and Putamen which is the Region of Interest (ROI) for this study. The area values were then fed to the ANN which is hypothesized to mimic the pattern recognition of a human observer. The simple but fast ANN built, could classify subjects with and without PD with an accuracy of $94 \%$, sensitivity of $100 \%$ and specificity of $88 \%$. Hence it can be inferred that the proposed system has the potential to be an effective way to aid the clinicians in the accurate diagnosis of Parkinson's disease.
\end{abstract}




\section{Acknowledgment}

First of all thanks to the Almighty for giving us the capability to conduct this research and finish our work successfully.

Moreover, we would all like to thank our thesis supervisor DR. Md. Ashraful Alam for guiding us all along and working as the main driving force whenever we lacked anything in our work. He kept his door open to us to assist with any problems, big or small, and enlightened us with his immense knowledge on our concerned topic.

Furthermore, sincere thanks to BRAC University for creating an entire thesis lab which is well facilitated with sufficient computers, fast internet and all equipment needed for our work. This lab provided all of our team members the opportunity to work together whenever we had to work as a team or work separately.

We would also like to thank the Parkinson's Progress Marker's Initiative (PPMI) for letting us use their data to conduct our study successfully.

Last but not the least, our sincere gratitude to every faculty members who assisted us in making ourselves capable enough throughout our university life and also to all the staffs of our university who are a part of creating a wonderful environment here that contributed to a good and healthy university life. 


\section{Contents}

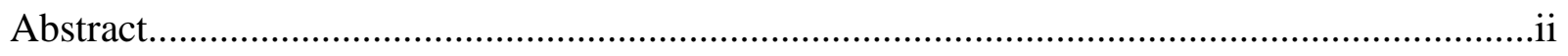

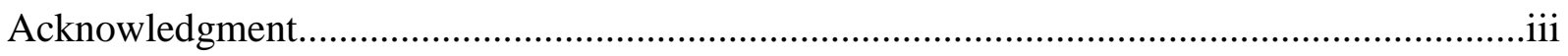

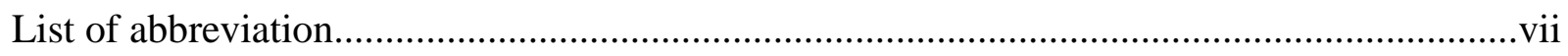

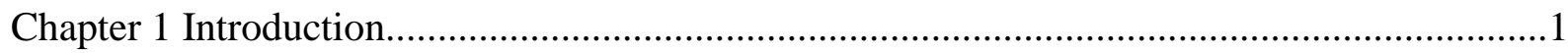

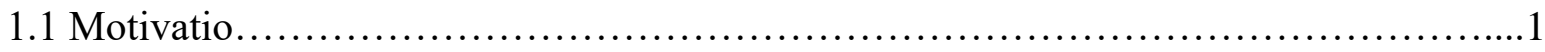

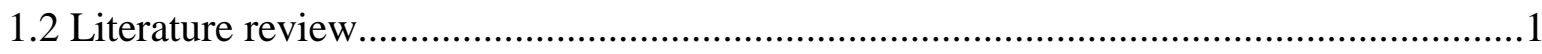

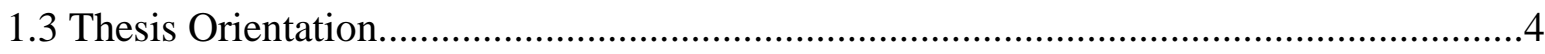

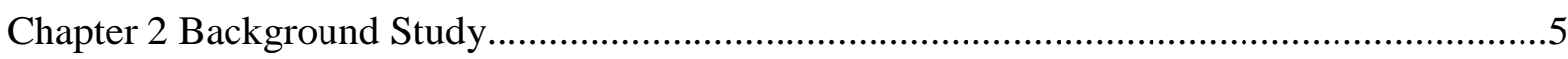

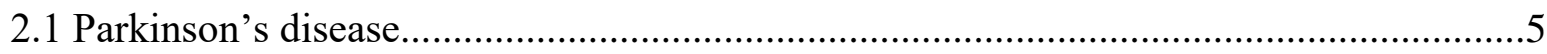

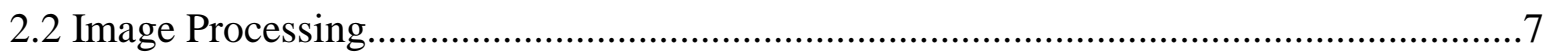

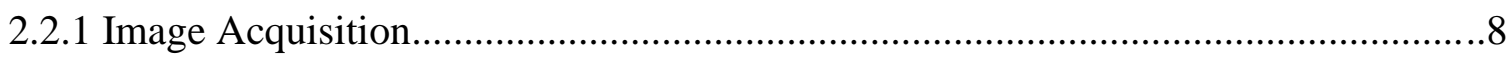

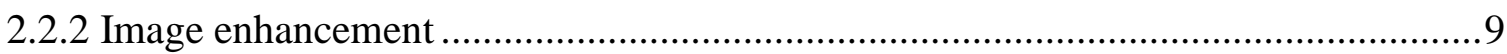

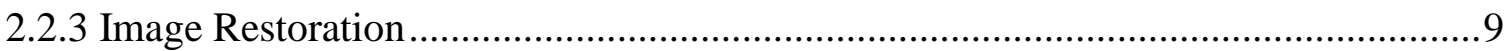

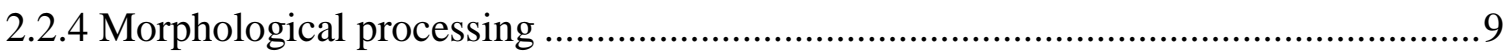

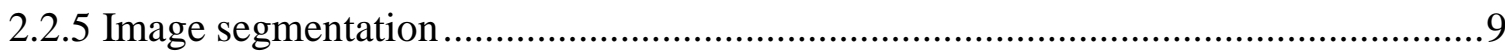

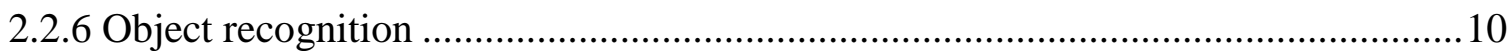

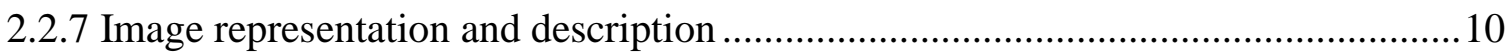

2.3 Image Processing in Biomedical field.....................................................................

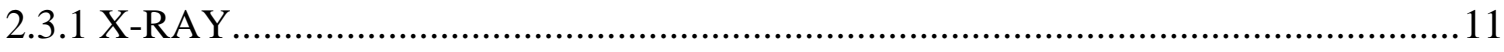

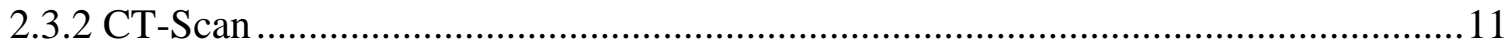

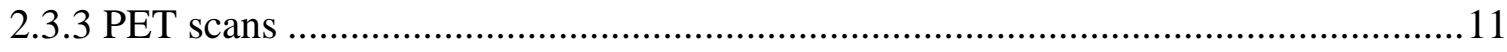

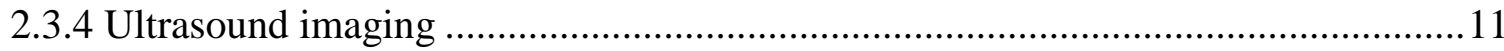

2.3.5 Magnetic Resonance Imaging MRI ..........................................................11

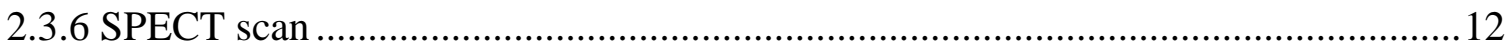

2.4 Techniques for processing SPECT images............................................................13

2.4.1 Hough Transformation: ................................................................................13

2.4.2 Sequential grass fire algorithm .....................................................................

2.4.3 Graph search algorithm .............................................................................14

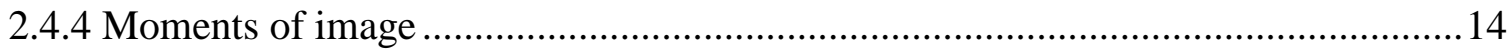

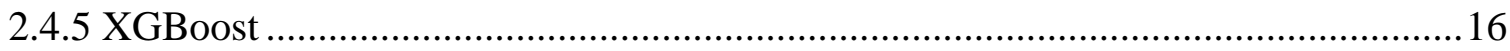

2.4.6 Logistic Regression ...................................................................................17

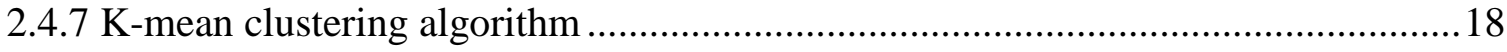

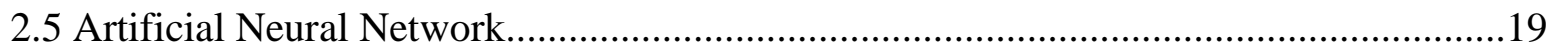




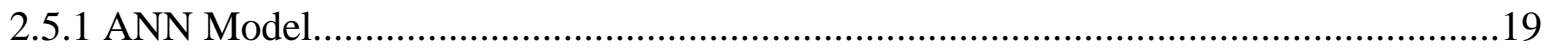

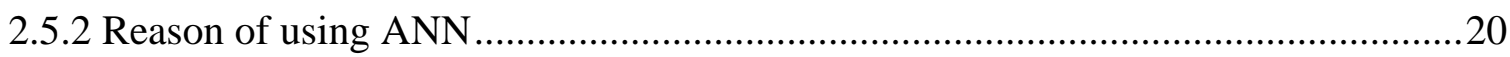

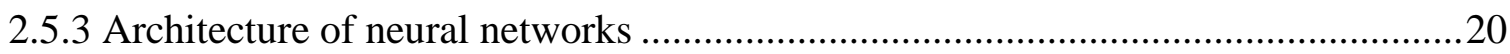

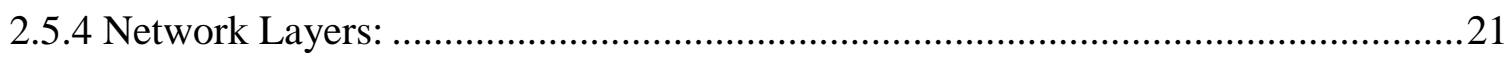

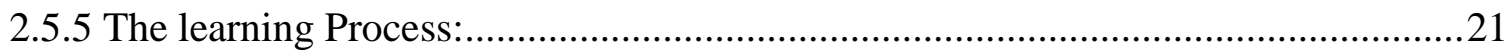

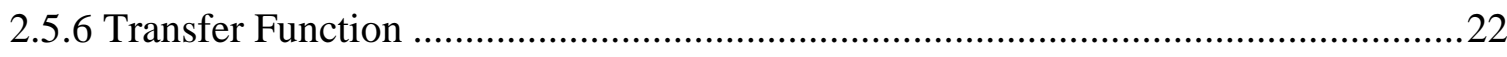

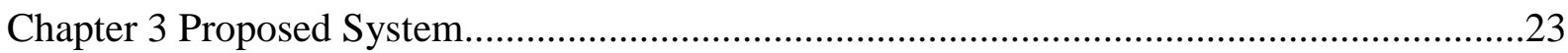

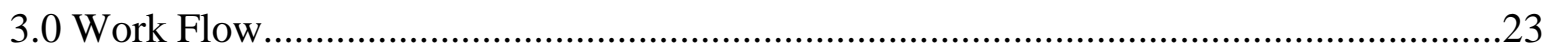

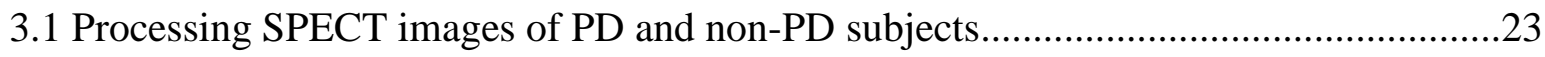

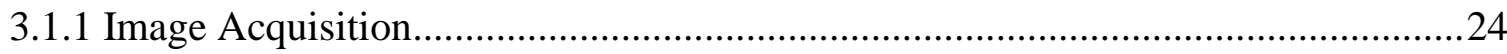

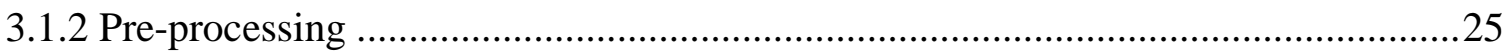

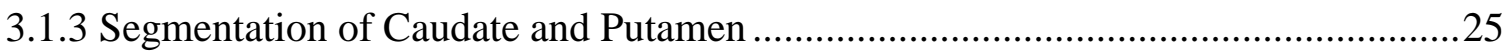

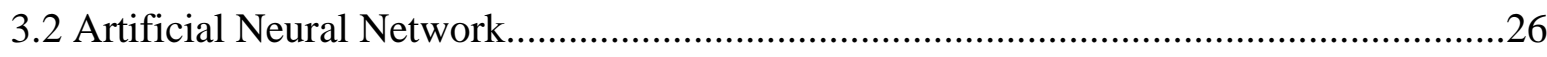

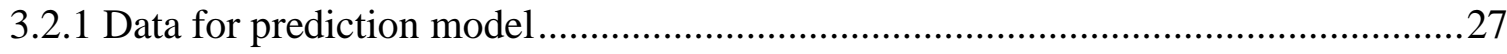

3.2.2 Choosing activation function ...........................................................................27

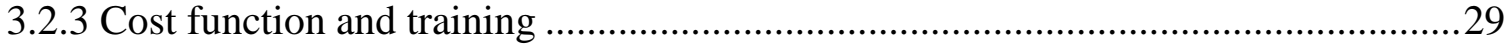

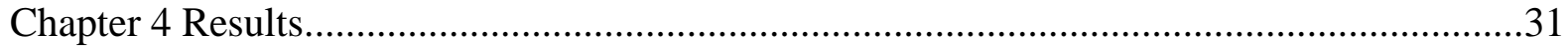

4.1 Image processing Result ........................................................................................ 31

4.1.1 Tabular Representation of area of ROI in PD subjects ............................................33

4.1.2 Tabular Representation of area or ROI in Non-PD subjects ....................................34

4.1.3 Comparison between Control and PD .................................................................. 35

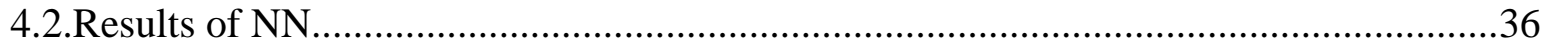

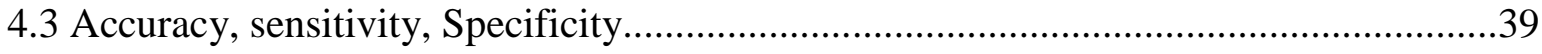

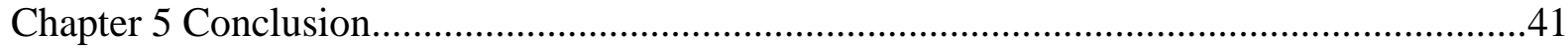

5.1 Future work

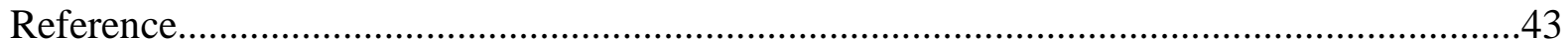




\section{Table of Figures}

1. Figure 1 The pathway of Dopamine in the Striatum region of Brain [10] ...................... 5

2. Figure 2Morphological Processing[11] ..................................................................... 9

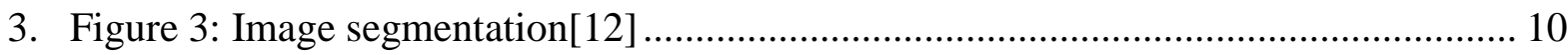

4. Figure 4: DaTSPECT images showing dopaminergic region on different subjects[13] .. 12

5. Figure 5: A binary image with white and black pixel[ [14] ........................................ 15

6. Figure 6: Features of XGBoost algorithm ............................................................ 16

7. Figure 7 How data are classifies using K-mean clustering. [15] ................................... 18

8. Figure 8 ANN model. ............................................................................................ 19

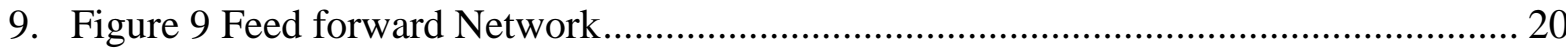

10. Figure 10 : Feedback Neural Network................................................................. 21

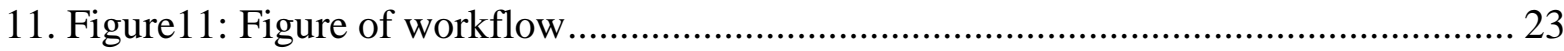

12. Figure 12: Block Diagram of Detection of PD using image processing....................... 24

13. Figure 13: Binary image before(left) and after(right) sharpening ................................ 25

14. Figure 14: The boundary of ROI detected with corresponding binarized images ........... 26

15. Figure 15: Sigmoid curve and Differential of Sigmoid Curve ..................................... 28

16. Figure 16 :Changes in cost function for individual training ...................................... 29

17. Figure 17 Results with test subject (in gray) in predicted area .................................... 30

18. Figure 18 Area of Putamen and Caudate detection of non PD patient .......................... 31

19. Figure 19: Area of Putamen and Caudate detection of patient with Parkinson's disease. 31

20. Figure 20: Left and Right area comparison of patients with Parkinson's...................... 32

21. Figure 21: Left and Right area comparison of patients without Parkinson's disease ....... 33

22. Figure 22: Comparison between sum of area right and left of the selected regions ......... 35

23. Figure 23: Results for non-PD subjects accurately predicted by ANN ........................ 39

24. Figure 24 Results showing PD subjects accurately predicted by ANN ........................ 39

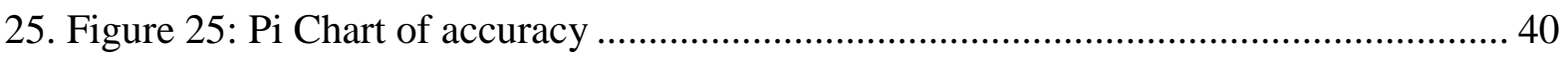


List of abbreviation

DAT Dopamine Transporter

SPECT Single Photon Emission Computed Tomography

PD Parkinson's Disease

CNN Convolutional Neural Network

ANN Artificial Neural Network

SWEDD Scans Without Evidence of Dopaminergic Deficit

ROI Region of Interest 


\section{Chapter 1}

\section{Introduction}

\subsection{Motivation}

Parkinson's disease (PD) is a long term degenerative disorder of the Central Nervous System which causes a diverse set of symptoms ranging from tremor to cognitive impairment, hallucination, dementia, sleep disorders etc. More than 10 million people in the world suffer with Parkinson's disease [1]. In Bangladesh, every year, approximately 1600 people die from Parkinson Disease. Till now there is no cure for PD [1]. However about ten years before the onset of tremor or motor symptoms, the dopaminergic neurons begin to change. Some premotor symptoms of Parkinson's disease (at an early stage) includes decrease in sense of smell, disorder in Rapid Eye Movement (REM) sleep, small handwriting, difficulty in moving etc. An early diagnosis of PD results in effective management and avoidance of unnecessary medical tests, therapies, costs, safety risks etc. Parkinson's at an early stage is most commonly detected using brain scans such as MRI, fMRI, SPECT, PET etc. Till today, in most clinics, these images are interpreted by clinicians; with added possibility of human error. In a study it was reported that the pooled accuracy of clinical diagnosis of Parkinson's disease is only 80.6\% [2]. This motivated us to come up with a new and simple approach to detect Parkinson's disease at an early stage automatically using Image Processing and Artificial Neural Network. Adapting this technology in hospitals or diagnostic centers can increase accuracy in diagnosis of Parkinson's and save money and time.

\subsection{Literature review}

Challa K.N.R. et al. have used different machine learning algorithms to improve models that already use important non motor symptoms of Parkinson such as rapid eye movement in sleep behavior disorder and olfactory loss along with Cerebrospinal Fluid measurements and dopamine transporter imaging to detect PD at early stage. They used four different machine learning classifier namely Multilayer Perception, Bayesian Network, Random Forest and Boosted Logistic Regression and tested the collected dataset on each four of them to find out the accuracy and precision of the classifier and plotted the ROI curve for each of the classifier. It was observed that though accuracy and area under ROI curve was almost same for every technique, 
Boosted Logistic Regression gave the highest performance with $97.16 \%$ accuracy and $98.9 \%$ percent area under ROI curve. Thus it was concluded that when Boosted Logistic Regression is used a machine learning technique along with other non-motor symptoms in detecting PD then a higher performance can be expected [3].

Choi H. et al. established a model which can rectify the human error where a patient is diagnosed clinically as PD but after performing visual analysis of FP-CIT SPECT clearly show no evidence of dopaminergic deficiency. Though dopamine transporter imaging (DAT) successfully detect dopamine deficiency but still sometimes it is inconsistent due to high sensitivity (67\%) and low specificity (67\%) in patients at early stage of PD. Hence the objective of their work is using deep learning based model in creating an automated FP-CIT SPECT interpretation system that can differentiate PD patients from patients with no clear signs of PD. For interpretation of these images $\mathrm{CNN}$ or deep convolutional neural network was used which learn image features from each set of training data to produce two nodes- one for PD and one for normal control. Moreover these models will separate SWEDD patients from patients wrongly clinically diagnosed as PD [4].

Rahmim A. et al. work was to show that whether longitudinal SPECT images in addition to normal imaging and non-imaging processes can play any role in the improvement of detecting PD at early stage. In order to do that image analysis was performed which included automatic region-of-interest extraction from MRI images, registration of SPECT images onto corresponding MRI images and extraction of radiomic features from caudate, putamen and ventral striatum of DAT SPECT images. After performing random forest analysis it was demonstrated that longitudinal DAT SPECT images along with random forest increase PD detection outcome [5].

Paul D Acton and Andrew Newberg used artificial neural network to imitate human pattern recognition skill that are able to diagnose and show similar results as conventional ROI analysis. The ANN was first trained with known dataset where 46 pixel values of striatum were passed into the input node which in turn was passed into the hidden layer. The hidden nodes gave final result as two end nodes, one of which indicated PD patient and one as normal. After these unknown dataset was applied on the ANN which resulted into an accuracy of $94.4 \%$ which is the 
highest of other methods which are Mean ROI, Best ROI, Mean observer and Best Observer included in the particular test [6].

To develop a model for early PD detection Prashanth R. et al. used SBR or striatal binding ratio values from I-Ioflupane SPECT scans. For building these particular model support vector machine (SVM) and regression model (RM) has been used. For SBR values the four striatal regions namely left and right caudate and left and right putamen is used. Comparing the two models used, it was observed that SVM when used with RBF kernel and prognostic model based on multivariate regression model both give high accuracy for detecting early PD and also differentiating early PD from SWEDD. In another work of the same authors, the respective authors developed a classification model to separate early PD patients from normal or SWEDD patients by SPECT scanning data of normal patients, PD patients and SWEDD patients to see which region of the scan shows most high activity. The classification model is created based on feature extraction after normalizing and segmenting data. The feature extraction has been performed through two processes. One is analyzing the shape of these regions which gave them 16 features and another is fitting a cubic surface to observe intensity on those particular regions which gave them 14 features. Along with this, the respective features are also compared with SBR based feature using Random Forests technique [7].

Oliveira F.P.M. et al. worked in extracting several features from FP-CIT SPECT brain images and tested them both individually and combinedly to test what works best for building a robust model that will assist in clinical decision of PD. To do this, three approaches were used from the sector of machine learning, namely - Logistic Regression, k-nearest neighbor (k-NN) and support vector machines (SVM). Altogether seven features were extracted of which five were uptake feature of striatum related feature and two were length and volume of striatum related feature. They concluded after experimenting that all the features when taken together into consideration gave more accurate results rather than taking any one feature but the accuracy was not due to the classifier used but rather on the specific feature that was selected [8].

IIIan I.A. et al. made an approach to computer aided diagnosis for developing fast, automatic and robust assistance techniques in detecting Parkinson early which will eliminate any unnecessary time consumed. They compared the performance of SVM classification with other classifiers and observed that amongst the available classifiers for performing this task SVM 
classification along with normalization gives highest result. It also evaluates the impact caused by different elements used for designing CAD. To conclude they stated that their work opened the possibility of applying different optimized algorithm to improve CAD performance along with SVM classifier [9].

\subsection{Thesis Orientation}

In the rest of the thesis, Chapter 2 discusses Parkinson's disease, the fundamentals of image processing, and Artificial Neural Network; Chapter 3 presents the proposed model of the research; Chapter 4 demonstrates the results found in our research; and Chapter 5 concludes the thesis. 


\section{Chapter 2 \\ Background Study}

\subsection{Parkinson's disease}

Parkinson's disease a long term degenerative disorder of the central nervous system that affects the motor control of a patient by affecting predominately dopamine producing ("dopaminergic") neurons in a specific area of the brain called substantia nigra [10]. The main problem in detecting the disease timely is the visible symptoms appear mostly at the later stage where cure no longer becomes possible. There is no correct reason proved yet that results to cause of Parkinson's, hence scientists are still conducting extensive research to find out its exact cause. Though some abnormal genes that becomes prominent due to aging seems to lead to Parkinson's in some people but there is no evidence to proof this.

Though there are a couple of procedures for early Parkinson's detection, Dopamine transporter single-photon emission computed tomography can be used to effectively diagnosis Parkinson's by detecting amount of dopamine deficiency in the concerned patient's brain cell at a considerable early stage. The pathway of Dopamine in the Striatum region is shown is fig 1.

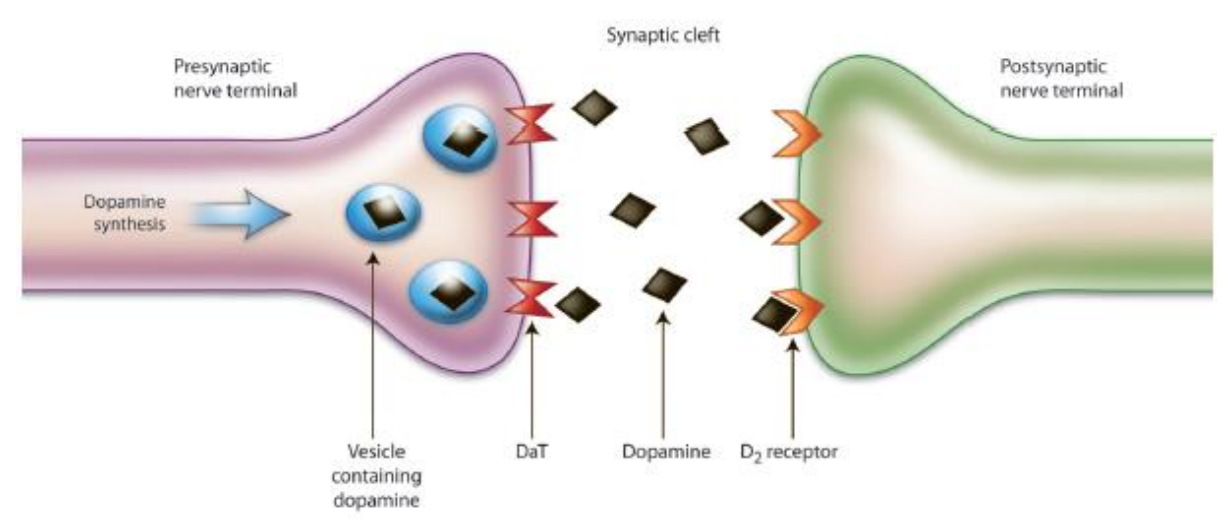

Figure 1 The pathway of Dopamine in the Striatum region of Brain [10]

No two patients will express symptoms of Parkinson's the same way, it is essential to take some common early symptoms through the five following known stages of PD into consideration and run diagnosis to confirm whether the concerned patient is suffering from PD or not so that treatments can be started as early as possible. 
The typical stages of Parkinson's disease are described below.

\section{- Stage one:}

At this stage no prominently visible symptoms appear that interfere daily activities to an extent where family members worry about. Slight tremor on any side of the body appears which in most cases is thought to be a sign of aging. Sleep disorder, smaller handwriting can also be symptoms.

\section{- Stage two:}

Symptoms shown in stage one starts to worsen. Tremor, rigidity and other motor symptoms affect all over the body. Though a person is able to carry out his daily activities at this stage but the activity duration becomes delayed.

\section{- Stage three:}

At this mid stage, loss of balance, slowness of movements of different parts of body and falling becomes very frequent. Patient starts the need of another person for feeding and dressing.

\section{- Stage four:}

At this stage assistance is required for accomplishing every daily activity. The patient is only able to stand alone.

\section{- Stage Five:}

This is the most serious stage where the patient loses ability to walk or do anything even with assistance. Severe stiffness in the leg make the person to be totally bedridden or in wheelchair and around the clock nursing is required.

Parkinson's disease is known to be a disease which shows considerable motor symptoms but recent researches suggests that PD shows many non-motor symptoms which are frequently misdiagnosed as signs of aging. If people would be more careful about the following non motor symptoms and visit nearest clinic for a diagnosis, a DAT SPECT imaging would have been able to classify the patient as PD or non PD. The most common non motor symptoms are quick fatigue, excessive sweating, constipation, early satiety, vision problems, sleep disorders, increase in dandruff, urinary urgency, olfactory loss, weight loss or gain.

At a later stage, when PD is confirmed in a patient, symptoms such as tremor at particular part of body becomes prevalent, movements become slower, muscles become rigid, posture and 
balance becomes impaired, speech and writing changes, suffers from loss of voluntary muscle movements, swallowing problems.

Full cure of Parkinson's disease is practically impossible but if gradually people become aware

of the symptoms that arise long before getting to the stage where no cure is possible then after correct detection, the concerned patient can undergo treatments that will save the patient from a lot of symptoms and ultimately prevent him from being totally dependent upon others for daily activities.

\subsection{Image Processing}

Image processing means systematic analysis of an image or the application of various algorithms to an image data in order to enhance the image and extract required information from the particular image. Image is actually the visual representation of any two or three dimensional Scene. It can be thought of like an array where the pixels are arranged in columns and rows. Whenever there is an image, there comes image processing for the sake of using that image in modern technology. This processing can be thought like a signal processing where the input is an image and the output is either an image or its associated features.

The main steps associated with any sort of image processing are as follows:

1. Import the input image through acquisition tools

2. Analyze and manipulate the image

3. Receive the output image or the result analysis of the input image.

The first use of image processing can be dated back to the early 1920s where images were coded in the receiving end of a submarine cable and those images were reconstructed at the receiving end by a telegraph printer. Gradually this process was highly improved and applied to a number of fields like agriculture, food industry, medical treatment, robot vision, digital cinema, remote sensing, etc.

The methods that are used for image processing are digital image processing and analog image processing. Analog image processing are used for hard copies like printouts and photographs and on the other hand digital image processing involves analyzing image in digitalized way using Computer or other digital device.

Amongst several uses of image processing in today's world, removing straight lines, separating aggregate of objects, detecting license plate, scanning whiteboard contents, detecting texts in 
still images, enhancing x-ray images, removing pattern from scanned photos, extracting desired areas in Google map, extracting cells in nuclei are a few to be mentioned.

\section{Digital Image Processing}

Digital image processing is the manipulation of images through a digital device such as computer. It focuses on building a computer system that runs an algorithm to take digital image as input and process that image to extract its features for output. The continuum from image processing to vision can be broken down to three phases, namely - low level image processing , mid-level image processing and high level image processing.

\section{- Low-level image processing}

Low-level image processing deals with image preprocessing through noise reduction, image enhancement and sharpening of the image.

- Mid-level image processing

Mid-level image processing deals with analyzing of the image through segmentation and classification of the image.

\section{- High-level image processing}

High-level image processing deals with vision of image through ensemble of recognized objects.

Stages of Digital Image Processing

In every digital image processing the following seven basic stages that are needed to be followed:

1. Image acquisition

2. Image enhancement

3. Image restoration

4. Morphological processing

5. Segmentation

6. Object Recognition

7. Representation and description

\subsubsection{Image Acquisition}

Image acquisition in digital image processing world is the process of retrieving a completely 
unprocessed image from a source for processing. It is the very first stage in the total digital image processing stage since no work can be proceeded without getting the required image first.

\subsubsection{Image enhancement}

In digital image processing image enhancement is to improve the image in a such a way that it can be easily interpreted to human viewers. Image enhancement can be again done in two ways , one is to through spatial domain methods that operate directly on pixels and frequency domain methods that operate on the Fourier transformation of the image.

\subsubsection{Image Restoration}

Image restoration in digital image processing is undoing the part of the image which contributes to the defect of the image. Degradation in image comes in many form like motion blur, misfocus of camera, noise. In case of blurring of images, image restoration is done by finding out the blur function and then to reverse it to remove blur. In case of noise, restoration can be done by sacrificing minimum amount of resolution.

\subsubsection{Morphological processing}

The technique of converting a gray scale image to binary image in which each pixel has strict value of either 0 or 1 when identification of objects within an image can be difficult is called morphological image processing. Different kinds of morphological processing in shown in fig 2 .

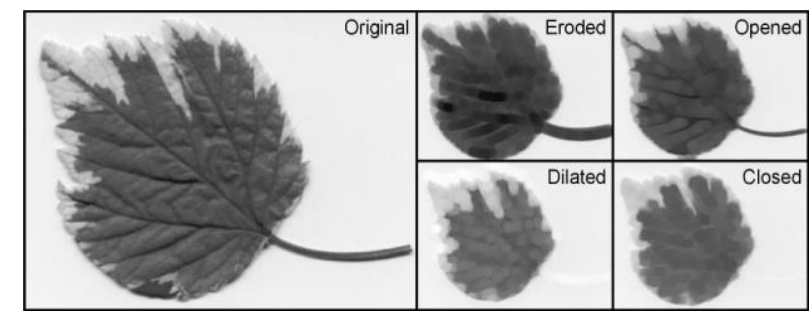

Figure 2: Morphological Processing[11]

\subsubsection{Image segmentation}

In digital image processing image segmentation is the process of partitioning a digital image into multiple segments or sets of pixels so that the image is changed to something more meaningful and easier to analyze. It is used for detecting boundaries or objects in a given image. In other words, what image segmentation does is assign label to every pixel so that pixels with same 
labels hold similar characteristics. Image segmentation is shown in fig 3.

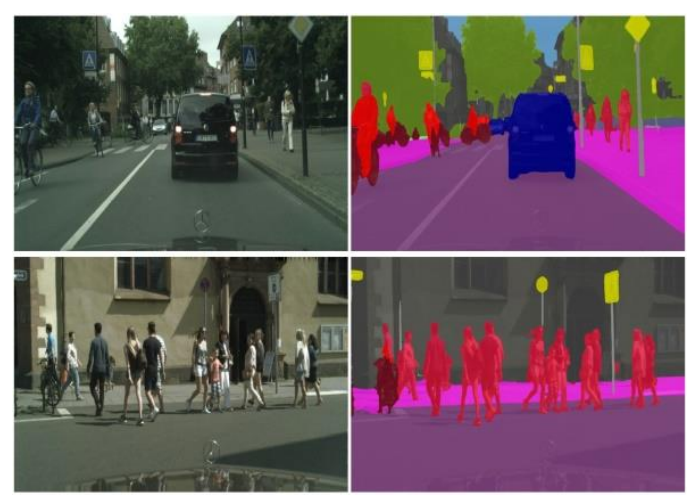

Figure 3: Image segmentation[12]

\subsubsection{Object recognition}

Object recognition in digital image processing is detecting a desired object from a given image. Various models such as feature extraction and machine learning models, deep learning models such as CNN, Bag-of-words models, gradient based and derivative based matching approaches, etc can be used for object recognition.

\subsubsection{Image representation and description}

This is the final step of digital image processing. After an image is successfully segmented and all its objects and background has been differentiated, then it is necessary to represent the objects properly with their exact features. Two main ways of representing a region of image is its external boundary and its internal characteristics. The region that is represented now should be made to describe using its features.

\subsection{Image Processing in Biomedical field}

Image processing evolved in the field of biomedicine as it has evolved in thousand other fields. Biomedical imaging methodically captures a biomedical image and process it both for diagnostic and therapeutic process. Doctors of today are able to detect several disease, come to major conclusions or take decisions from just seeing a processed image. Image processing allows humans to identify and analyze even the smallest things that might not have been visible to human eye. Moreover through image processing techniques, diameter volume and other features of tumor can be found out, flow parameters of blood or other fluids in human body can be analyzed clearly. 
Biomedical imaging uses either x-rays(CT scans), sound(ultrasound), magnetism (MRI), radioactive pharmaceuticals (nuclear medicine: SPECT, PET) or light (endoscopy, OCT) to asses the current condition of any inner part of human body.

\subsubsection{X-RAY}

X-ray was first invented in 1895 and is considered the first processed image and the initiative point behind sensors, instruments and software that are used to obtain biomedical imaging. In the beginning x-rays had many limitations to what it could reveal but gradually the quality of x-ray images improved.

\subsubsection{CT-Scan}

CT or CAT scans are special X-ray tests that produces cross-sectional images of the body using X-rays and a computer. It was developed independently by a British engineer named Sir Godfrey Hounsfield and Dr.Alan Cormack. These types of scans have improved patient's comfort as it can be done quickly.

\subsubsection{PET scans}

It is a radioactive tracer to measure metabolic changes, blood flow and oxygen use. It enables researchers to analyze for example the brain activity of a human during periods of depression based on the chemical activity of the brain.

\subsubsection{Ultrasound imaging}

Ultrasound imaging produces sound waves to produce pictures of the inside of the body through producing sound waves. It helps to diagnosis causes of any pain, swelling, infection inside the body, or condition of a baby inside a pregnant woman, delicate body parts of infants.

\subsubsection{Magnetic Resonance Imaging MRI}

MRI or magnetic resonance imaging uses a powerful magnetic field, radio waves and a computer to produce detailed pictures of inside the body. MRI safely monitors baby at the time of pregnancy. It is also used to diagnose variety of conditions within chest, abdomen, pelvis areas. 


\subsubsection{SPECT scan}

Firstly, single Photon Emission Computed Tomography or SPECT scan is a kind of nuclear medicine tomographic imaging technique that uses gamma rays and is able to provide true 3D information. It is used in biomedical sectors to observe the blood flow in tissues and organs. The integration of two technologies: computed tomography (CT) and a radioactive material (tracer) are used in SPECT scan. The tracer allows doctors to see the respective blood flow.

A patient is injected with radiolabeled chemical emitting gamma rays before performing SPECT scan. These rays are collected by computer to gather information and converts into two dimensional cross sectional area which can then be added together form a 3D image of the patient's brain. SPECT are more useful in brain injured brains than MRI or CT scanning because it is able to show even the reduced flow of blood in injured sites.

Now comes what Dopamine Transporter or DAT SPECT imaging is. ${ }^{123}$ I-Ioflupane is a SPECT molecular imaging agent for imaging dopamine transporter (DAT) in brain cells. In human brain the dopamine transport can be found highest in the stratium regions. These transporters caries dopamine from the cleft of synaptic region to presynaptic axions for either reuse or degradation. This degeneration results into lower density of dopamine transporters in the stratium regions. DAT SPECT imaging is an excellent way to detect the amount of dopamine loss and thus contributes a lot in detecting whether a patient has PD or not. DaTSPECT scans in various stages of PD and healthy subjects are show in fig 4.

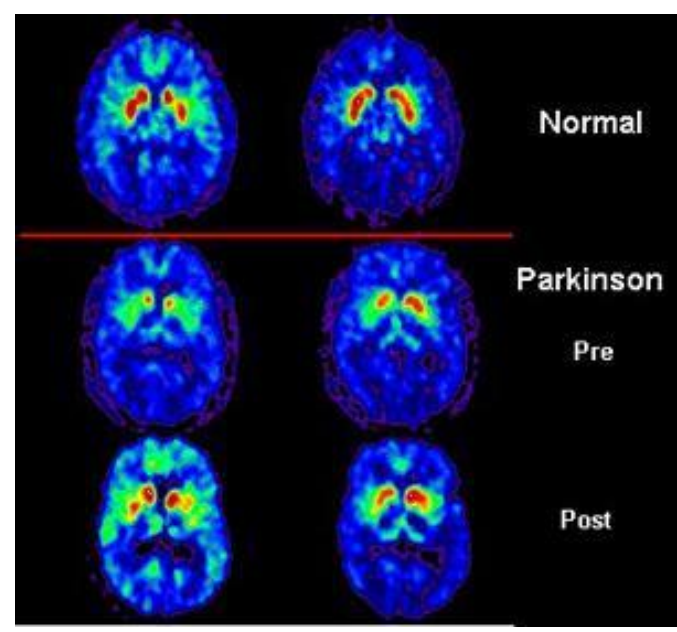

Figure 4: DaTSPECT images showing dopaminergic region on different subjects[13] 


\subsection{Techniques for processing SPECT images}

\subsubsection{Hough Transformation:}

Hough transformation is a technique for extracting a particular geometric feature of an image. Hough transformation was originally developed for detecting straight lines, it was then modified for detecting arbitrary shapes like circle, ellipses, etc. Though straight lines can be describes by the equation

$$
y=m x+c
$$

but some problem arise with these equations when the line is vertical. Hence for Hough transformation the following equation is used

$$
r=x \cos \theta+y \sin \theta
$$

which is then modified to look like (1) for convenience in (3).

$$
\Rightarrow \mathrm{y}=-\frac{\cos \theta}{\sin \theta} \cdot x+\frac{r}{\sin \theta}
$$

The advantage of this equation is every lines can be represented with this as long $\theta \in[0,180]$ and $\mathrm{r} \in($ or $\theta \in[0,360]$ and $\mathrm{r} \geq \theta$ ) where $\theta$ is the angle of the line and $\mathrm{r}$ is the distance of the line from the origin.

1. Reduce the image to a set of edges which serves as input image for Hough transformation.

2. The edges are detected mostly using Canny edge detector.

3. Map edge points in Hough space and store in an accumulator.

4. Through thresholding and other constraints the accumulator is searched for any infinite line

5. Infinite lines are converted to finite lines.

6. Finite lines superimposed on original image.

Hough transformation is a voting technique that can be used to answer possible questions like given points that belong to a line, what is the line, or how many lines are there, or which points belong to which lines. 


\subsubsection{Sequential grass fire algorithm}

Sequential grass fire algorithm scans image from top left to bottom right to find the object image. Once found it labels the object of the output as 1 and in input image it sets the same pixel as 0 . Next it checks the neighbor from four to eight pixels depending upon how many of the pixels are connected to the present pixel and search if it as an object pixel. Now if any of the pixel is an object pixel then the output pixel image is set to 1 and the input pixel is set to 0 . These setting of 0 are called setting fire. These pixels are send to a list where each of the pixels is investigated following the scan path until it meets the next object pixel which is labeled as 2.The result of this kind of setting fire is getting a skeleton of the required part of the image in the pixels where the fire is set.

The main result that grassfire provides is it forms a skeleton in the region where the fire meets. This skeleton provides important description about images such finding its subparts, depressions and protrusions. It also provide interior region of shape of a boundary. Also, the skeleton that it provides is invertible. When the medial axis or skeleton of an image is given, the original image can be found by moving with the waveforms outwards.

Grass-Fire algorithm is used in blob detection, to find interconnected pixels in image processing field.

\subsubsection{Graph search algorithm}

This algorithm provides ways to search for a specific destination point from the starting point of a given graph. A graph is a structure which consists a set of $\mathrm{N}$ nodes and a set of $\mathrm{A}$ ordered pairs of nodes which are called edges or arcs. To avoid visiting the same visited nodes again and again , the already visited nodes are colored or marked as visitation state. The two most common form of graph search algorithms are breadth first search (BFS) where all the siblings nodes are searched before searching the child nodes and depth first search (DFS) algorithm where it traverses to the deepest node of the root node, then backtracks and searches all other nodes in the same process. Graph search algorithm can be used to find number of connected components.

\subsubsection{Moments of image}

An image moment is a particular number which defines an image and is constructed from a spatial random variable. It can be calculated after segmenting the image to extract features like 
area, centroid and orientation. There are two orders of masses in a particular moment. The first gives the center of mass of the image defining the pixel density and the second order shows how the mass varies around the center. An image function is a term that refers to any piece-wise continuous real function $\mathrm{f}(\mathrm{x}, \mathrm{y})$ of two variables defined on a compact support $\mathrm{D}$ and having a finite nonzero integral. For a continuous function $f(x, y)$ the general form of a moment of order $(\mathrm{p}+\mathrm{q})$ is

$$
\mathrm{M}_{\mathrm{pq}}=\int_{-\infty}^{\infty} \int_{-\infty}^{\infty} x^{p} y^{q} f(x, y) d x d y ; \mathrm{p}, \mathrm{q}=0,1,2 \ldots \infty
$$

where $\mathrm{M}_{\mathrm{pq}}$ is moment of image of function of two variable $\mathrm{x}$ and $\mathrm{y}$ of the order $\mathrm{p}$ and $\mathrm{q}$.

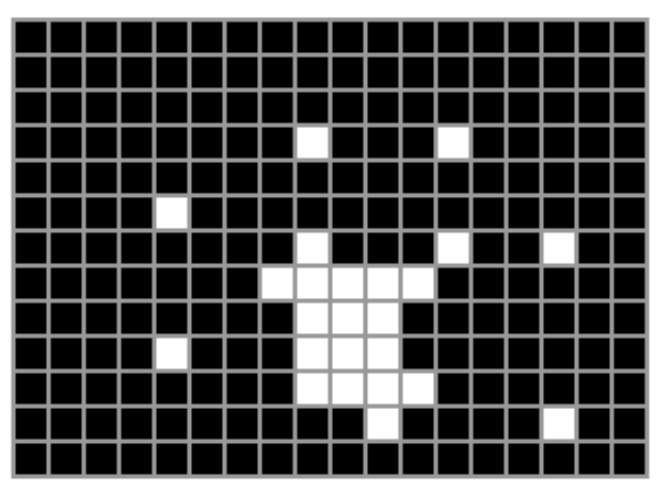

Figure 5: A binary image with white and black pixel[14]

We can take the above diagram (fig 5) and calculate its area from its moment. Calculating area is just one of many things that can be calculated from moment of image.

$$
\mathbf{M}_{\mathrm{pq}}=\sum_{x=1}^{W} \sum_{y=1}^{H} x^{p} y^{q} f(x, y)
$$

where $f(x, y)$ represents the image with width $W$ and height $H$. 
For the area of an image of zero ${ }^{\text {th }}$ order, $\mathrm{p}=0$ and $\mathrm{q}=0$. Thus $x^{p} y^{q}$ becomes $x^{0} y^{0}$ which do not have any effect upon the formula so they can removed and the formula can be simplified to the following.

$$
\mathbf{M}_{00}=\sum \sum f(x, y)
$$

Binary image comprises of two values ' 0 ' or ' 1 '. For every pixel that is white, 1 is added to the moment which results to area of the image.

\subsubsection{XGBoost}

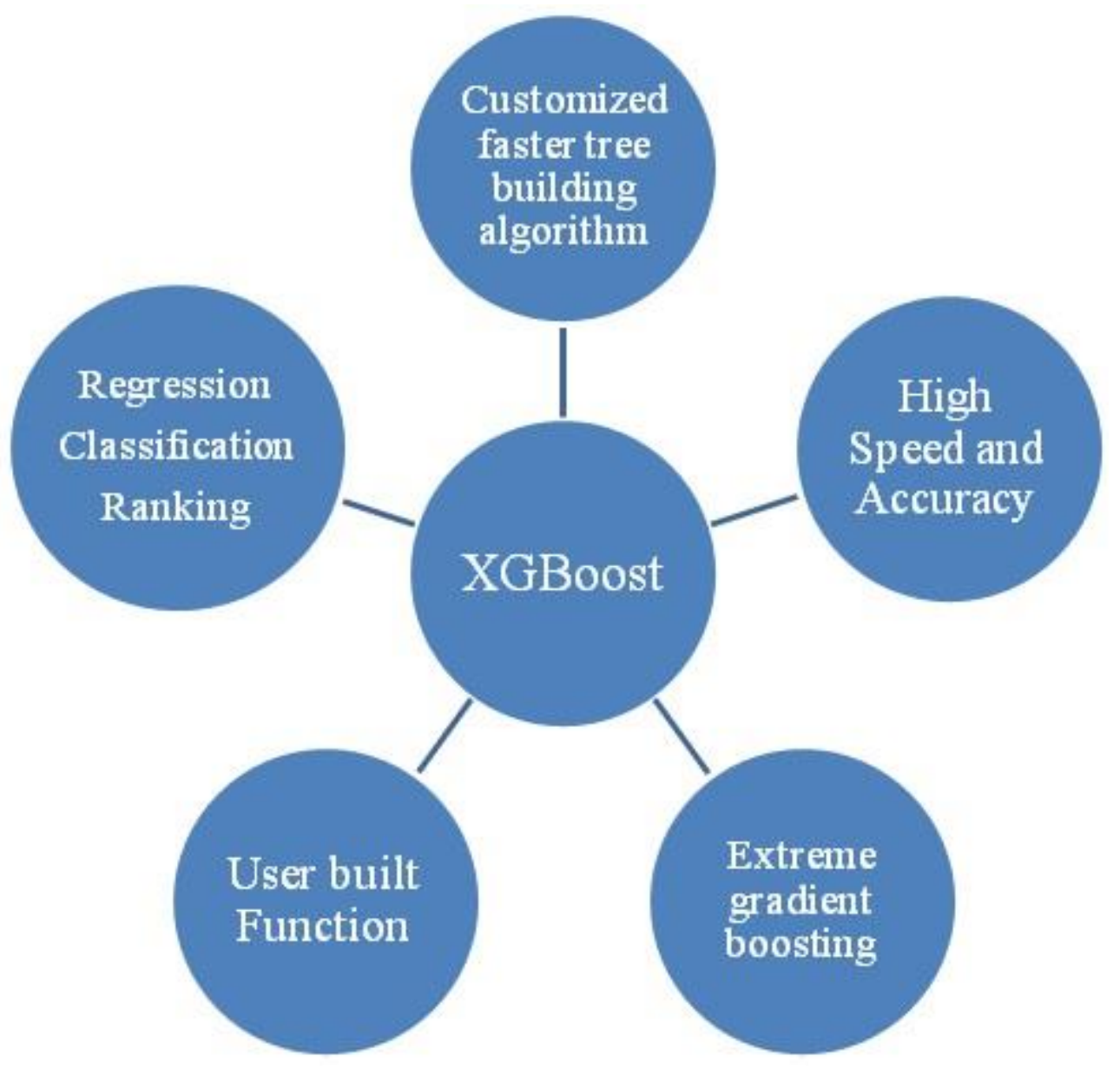

Figure 6: Features of XGBoost algorithm 
XGBoost, the abbreviated term of the algorithm known as the eXtreme Gradient Boosting,t is a highly flexible and versatile tool that exceeded computing power of boosted trees algorithms and engineered specifically to utilize the whole memory and hardware resources that are used for tress boosting algorithms. Though Tianqi used it first in command line interfaces, it can be used after loading in various programming languages like Python, C++, R, Julia, Scala and Java. It can successfully work through most regression, classification and ranking problems and even with user built objective functions and robustly supports fast tuning and adding of regularization parameters which makes it different and superior than other libraries. The following benchmark done by Szilard Pafka shows how XGBoost outperforms other similar algorithms without compromising speed or accuracy.

\section{The working procedure of XGBoost:}

1. Firstly, boosting is a method that creates strong classifier from weak classifier by continuously adding models on top of each other one by one so that errors of previous model is corrected by the next one until the training model is totally corrected or reproduced to a better one.

2. Next comes the gradient boosting part the same procedure of adding models is following but instead of putting a weight to the classifier after every step, it fits the new model to the left out part of previous model. Hence it is called gradient boosting since it updates previous models using gradient descent. Regression and classification problems both are supported by these..

3. Finally, XGBoost implements these same decision tree boosting with added speed and computational accuracy.

\subsubsection{Logistic Regression}

Logistic regression is a regression model with a categorical dependent variable. It is a go to method of machine learning for binary classification problems. Mainly due to the problem of linear regression where output can be less than 0 and greater than 1 but result of probability result must be 0 to 1 , logistic regression turned out to be its solution.

This model can be of three types - binomial, ordinal, multinomial Binomial logistic regression always gives two output shown in the form of 0 or 1 , multinomial deals in cases where three or 
more than three output is expected but the outputs are not ordered and ordinal deals with three or more ordered outputs. Logistic regression is represented with the following equation

$$
y=e^{\frac{(b 0+b 1 x)}{1+e^{b 0+b 1 x}}}
$$

Where $\mathrm{y}$ is the predicted output, $\mathrm{b} 0$ is the bias or intercept term and b1 is the coefficient for the single input value $(\mathrm{x})$.

\subsubsection{K-mean clustering algorithm}

K-means clustering is a type of unsupervised machine learning method to classify a given unclassified set of data[15]. This algorithm follows a very simple way to classify a given set of data through a certain number of clusters which are denoted by $\mathrm{K}$.
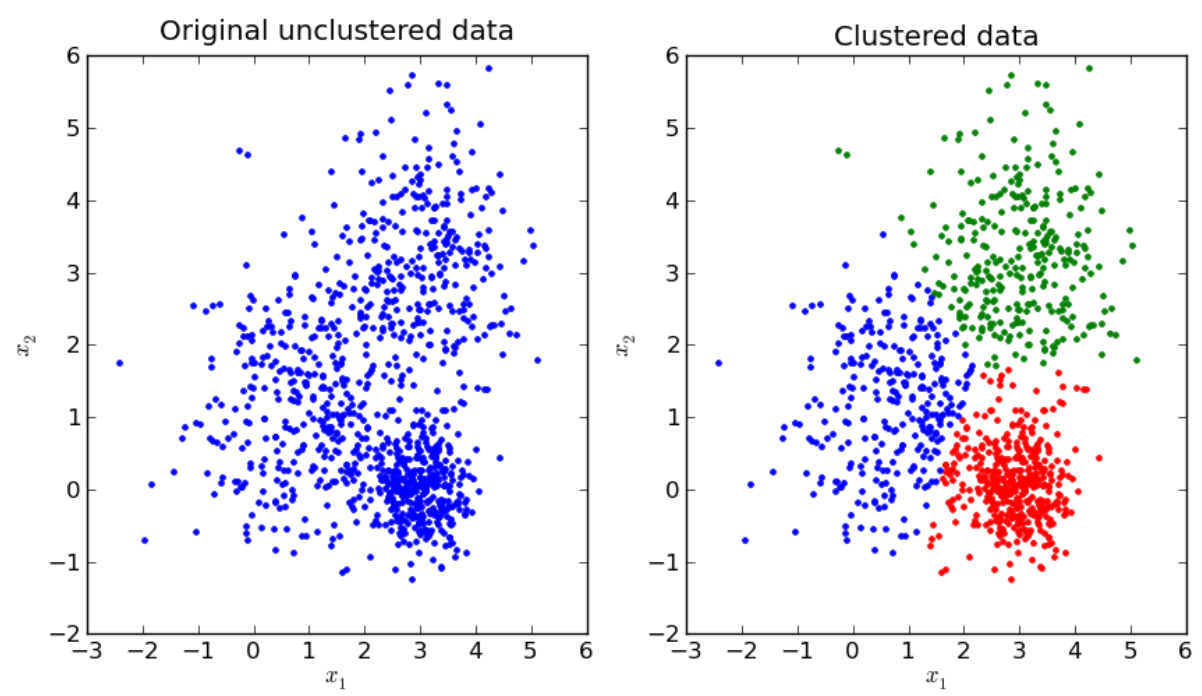

Figure 7 How data are classifies using K-mean clustering. [15]

The main step of this algorithm is properly defining $\mathrm{k}$ centers, one for each cluster and placing them as far as possible. If there is a given set of data points $X$ where $X=\{x 1, x 2, x 3, \ldots, x n\}$ and the centers of the data points are $\mathrm{V}$ where $\mathrm{V}=\{\mathrm{v} 1, \mathrm{v} 2, \mathrm{v} 3, \ldots \mathrm{vc}\}$, the algorithm follows the below steps.

1. It randomly selects centers of the clusters and denotes them as 'c'.

2. The distance between each data point and the cluster center is found out.

3. Find the minimum distance from cluster centers and assign a data point to this center. 
4. The new cluster center is recalculated using the following formula

$$
v_{i}=\left(\frac{1}{c_{i}}\right) \sum_{j=1}^{c_{i}} x_{i}
$$

5. Distance between newly obtained cluster centers and each data point recalculated.

6. If no data point is found then the algorithm stops here or else repeat 3.

The result of the algorithm provides centroids of the $\mathrm{K}$ clusters and also label for the training data.

\subsection{Artificial Neural Network}

\subsubsection{ANN Model}

Artificial Neural Network is an information processing system that is inspired from biological neural networks in animal brains. ANN consists of connected units called nodes. The connection between each node acts similar to a synapse in human brain to transmit signal. This structure of ANN is the key element of this paradigm.

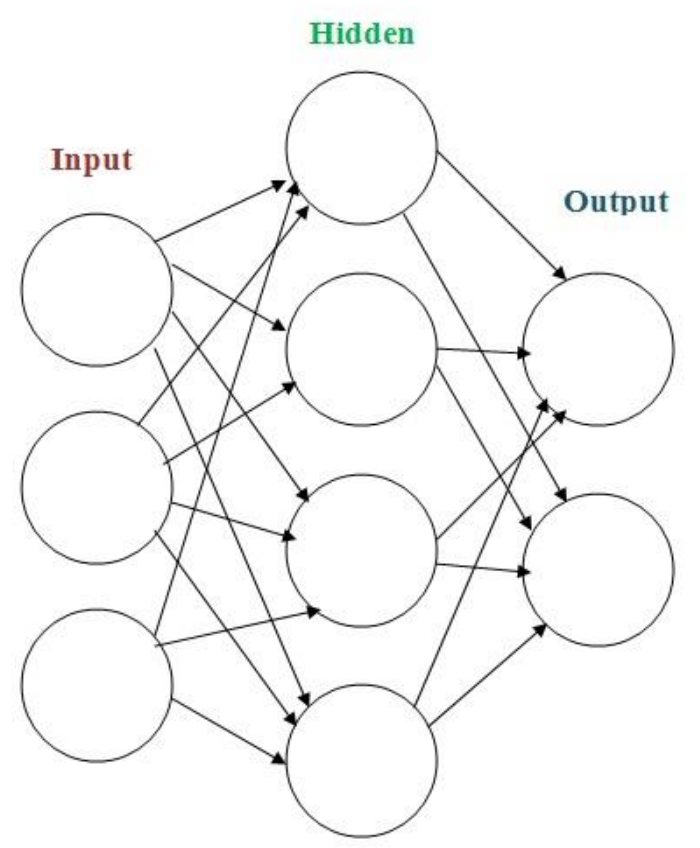

Figure 8 ANN model. 


\subsubsection{Reason of using ANN}

An Artificial Neural Network has an excellent ability to recognize patterns, extract meaning from a complex and imprecise set of data. A neural network can be thought as an "expert" in the task it is given to perform or data given to analyze. This expert can give decision given a new situation of interest and answer a "what-if" question.

Other advantages:

- Adaptive learning

- Self-organization

- Real-time operation

- Fault tolerance

\subsubsection{Architecture of neural networks}

\section{- Feed forward Network}

It allows signal to travel in one direction only, i.e. from input to output (as shown in fig 9). There is no feedback or loops. This type of network is mainly used in pattern recognition.

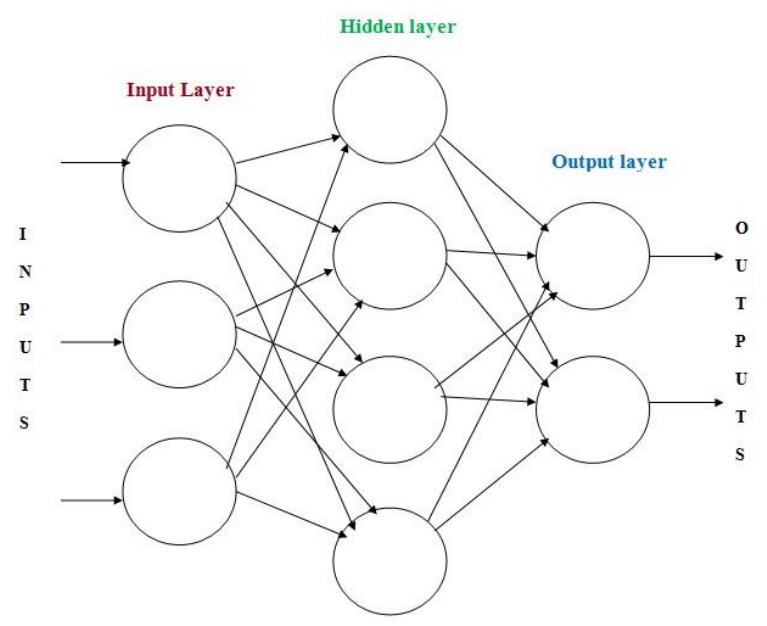

Figure 9: Feed forward Network

\section{- Feedback Networks}

Feedback network consists of loops, which allows signals to travel in both directions(as shown in fig 10). This network is dynamic and can be extremely complex. The states continuously change until they reach an equilibrium state. 


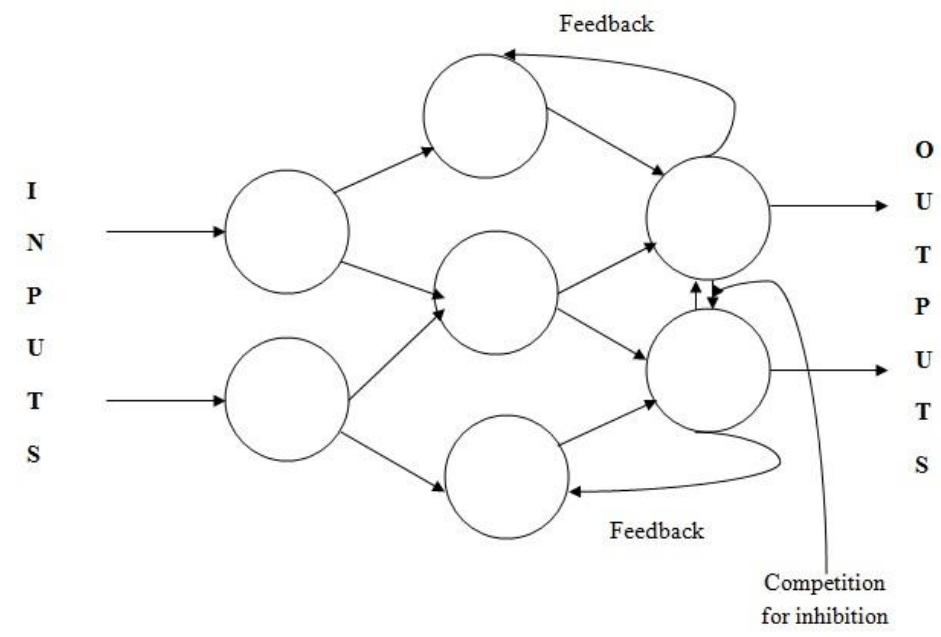

Figure 10 : Feedback Neural Network.

\subsubsection{Network Layers:}

Commonly consists for 3 layers:

- Input layer: raw information fed to the network

- Hidden layer: depends on the input and the weights of the connection

- Output layer: depends on the activity of the hidden layers and the weight between the hidden layer and the output layer.

\subsubsection{The learning Process:}

The information is stored in a weighted matrix called $\mathrm{W}$, the determination of weight is the process of learning. We can categorize this learning process into two major groups:

1. Fixed Network: in which the weight cannot be changed.

2. Adaptive network: are able to change their weight. The adaptive network can be further classified into two groups:

- Supervised learning: which includes an external teacher, i.e. a set of example pairs (x, y) where $\mathrm{x} \epsilon \mathrm{X}$ and $\mathrm{y} \epsilon \mathrm{Y}$, is needed to train the model to achieve an output for the function $f: \rightarrow \mathrm{XY}$ that matches the example in the allowed class of functions. The model creates a mapping inferred from the data. The cost function is the mismatch between the data and the mapping, commonly the mean-squared error, which tries to minimize the error 
between the network's output and the target value over all the example pairs. Task that fall under supervised learning is pattern recognition and regression.

- Unsupervised learning: No external teacher is needed and the decisions are made based on local information. Tasks that fall under this kind of learning is estimation problems, clustering, statistical distribution, compression and filtering.

\subsubsection{Transfer Function}

The functions followed by ANN falls under three categories:

Linear: the output activity is proportional to the total weight activity.

Threshold: The output depends on whether the total input in greater than or less than the threshold value

Sigmoid: the output varies continuously in a non-linear way as the input changes. They resemble actual neurons better than the linear and threshold functions. 


\section{Chapter 3}

\section{Proposed System}

The proposed system follows several steps. Firstly, the required data are collected from PPMI database. Then the images are preprocessed, and then the region of interest of our work is segmented from the processed image and detected. The area is calculated from the current image and is fed as the input of neural network. Now the prediction model is obtained in which the subjects are tested for acquiring the desired result that whether the patient has PD or is normal.

\subsection{Work Flow}

The work flow followed during the thesis is described in figure 11.

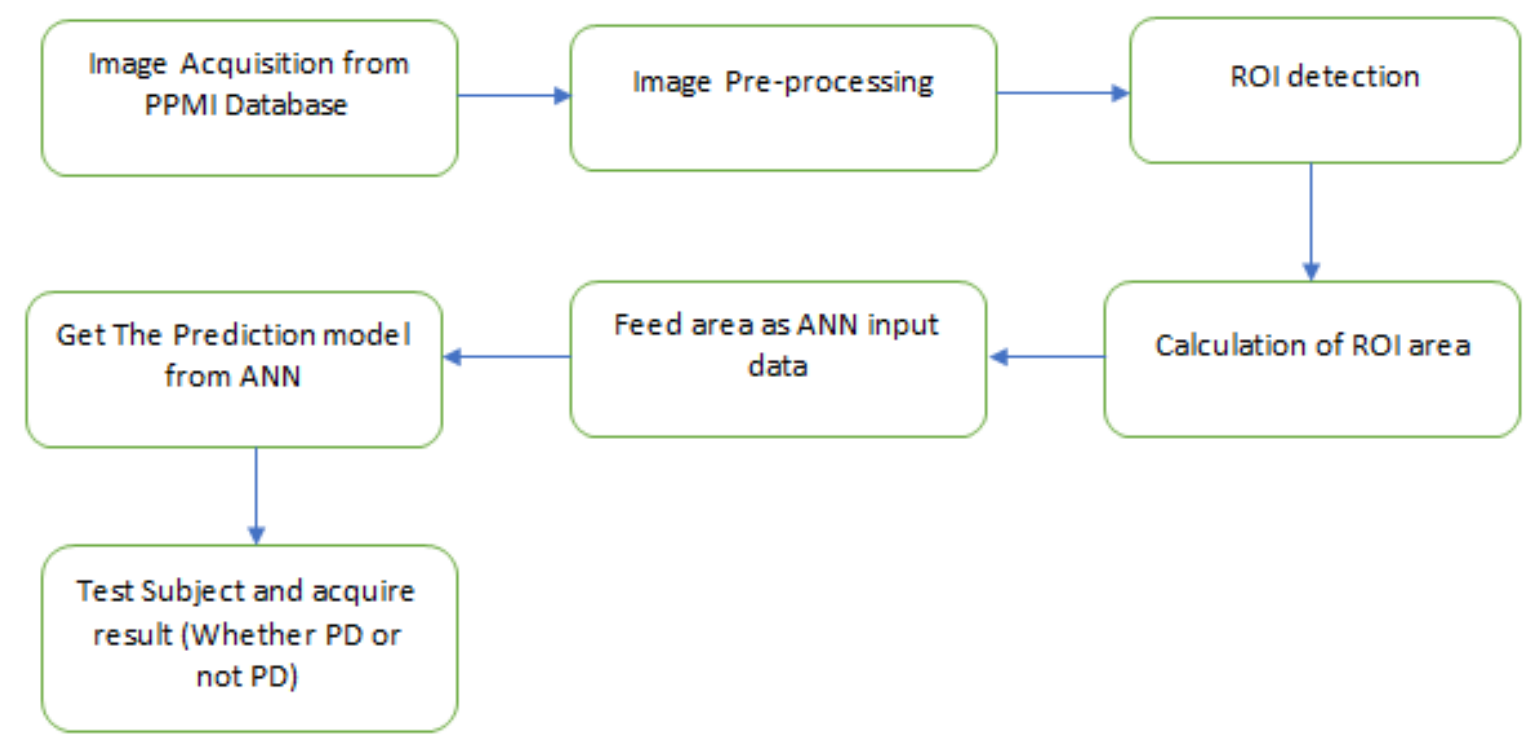

Figure 11: Figure of workflow

\subsection{Processing SPECT images of PD and non-PD subjects}

The application of image processing can be the crucial for the detection of Parkinson. In this section segmentation of Caudate and Putamen is done and areas of them is measured after being processed. The summary of image processing is described in fig 12. 


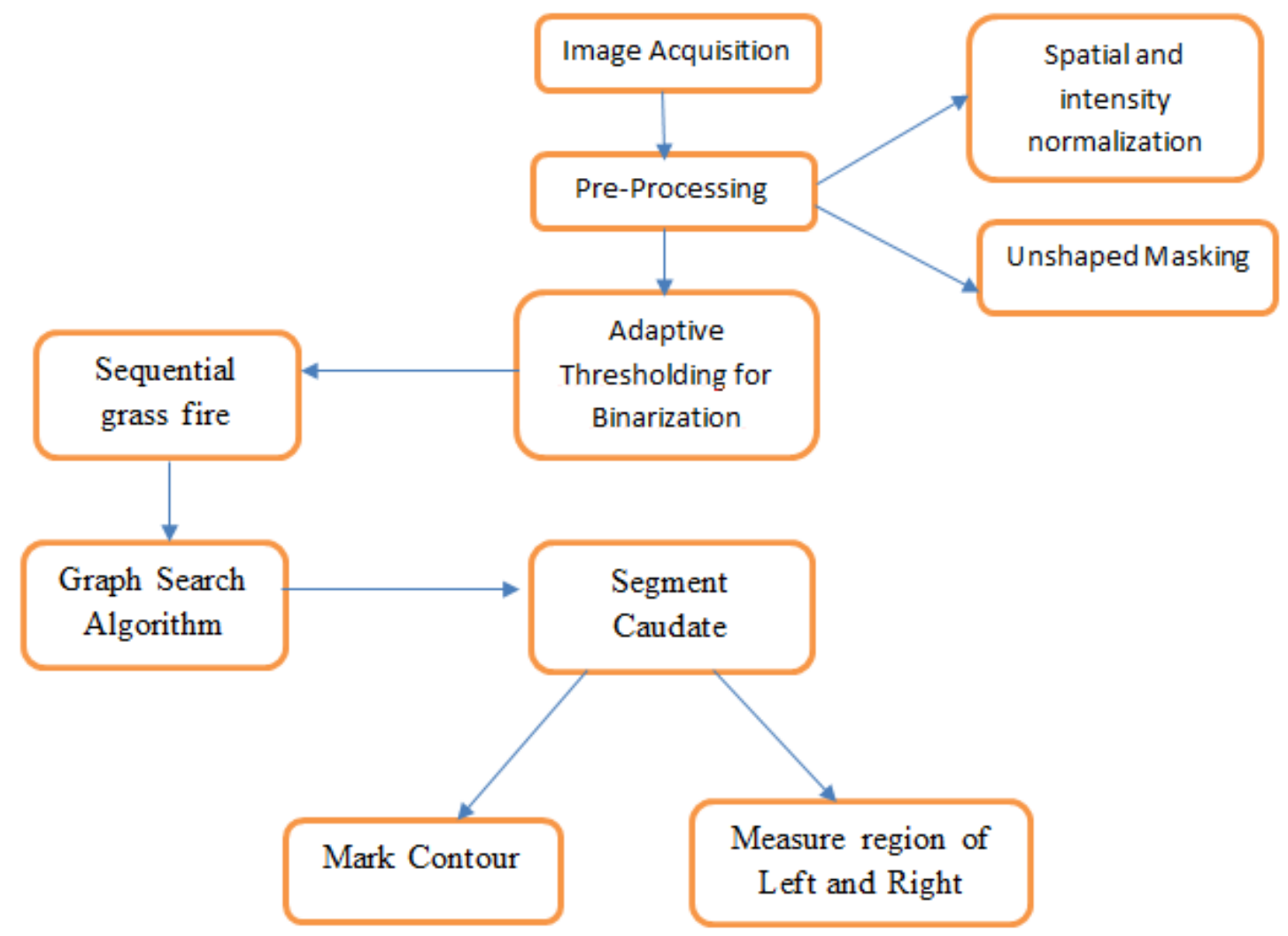

Figure 12: Block Diagram of Detection of PD using image processing

\subsubsection{Image Acquisition}

Data used for this study were obtained from Parkinson's Progress Marker's Initiative (PPMI) database [16]. It is a landmark study used to evaluate groups of people using advance imaging, biological sampling and clinical behavioral assessment to identify biomarkers for better treatment of Parkinson's disease. They have clinical sites in United States, Europe, Israel, and Australia. In order to gain access to their database one has to be a registered user. We have downloaded about 200 DaTSCAN SPECT images from their database of both healthy normal (control) and PD. Since the aim is to detect Parkinson's disease at an early stage, we have chosen subjects in early stage only (prodromal stage and early visits). 


\subsubsection{Pre-processing}

The preprocessing is done in two steps: spatial and intensity normalization. PPMI reconstructs the raw projection data and attenuates them. They also perform the spatial normalization for maintaining consistency in orientation (so that location in one subject's scan corresponds to another subject's scan). One additional step can be performed; a binary mask application, which reduces the number of voxels used in the subsequent analysis. Each scan consists of 91 axial slices from top to bottom of the head. We have selected slice in the range 39-42 as the Putamen and Caudate region is most visible in this range( slice 40 in most cases). It's only needed to be sharpened based on unsharped masking with standard deviation of the Gaussian lowpass filter value 7 and Strength of the sharpening effect value 2 (shown in figure 13).
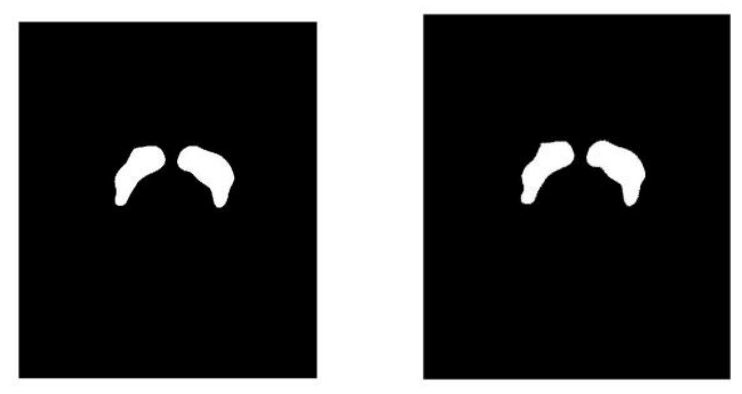

Figure 13: Binary image before (left) and after (right) sharpening

\subsubsection{Segmentation of Caudate and Putamen}

Segmentation of the caudate and Putamen is applied in order to calculate the stage of PD by measuring the size of both left and right using adaptive thresholding[17], Heuristic Graph Search algorithm[18] and Sequential Grass-Fire algorithm [19]. As the background is not relatively uniform and has a large variation in the intensity, so the adaptive aka dynamic thresholding is applied with the value between 0.52 to 0.71 for binarziation and edge detection. Each threshold value is the weighted mean of the local neighborhood minus an offset value. As a result, it segments both caudate and Putamen in binary form. Afterwards, for noise cancelation [20-21], adaptive median filtering is used as it does not erode away edges or other small structure in the image that filters and smooth the data while keeping the small and sharp details. Moreover, to detect connected neighbor pixels, Sequential grass fire algorithm ${ }^{\text {[19] }}$ is applied. Scanning starts from top left to bottom right to find non-zero pixels and once found, it labels the object of the output as 1 and in input image it sets the same pixel as 0 . It checks the 
neighbor from four to eight pixels depending upon how many of the pixels are connected to the present pixel and search if it as an object pixel. After that, the boundary pixels of left and right caudate are acquired and by changing the value with a RGB color like $(255,0$, 0: red), the boundaries are marked for visualization (as shown in fig 14). To find the area inside the bounded region, heuristic graph search algorithm is used. The connected pixels are summed as area and stored for further analysis [22-23].
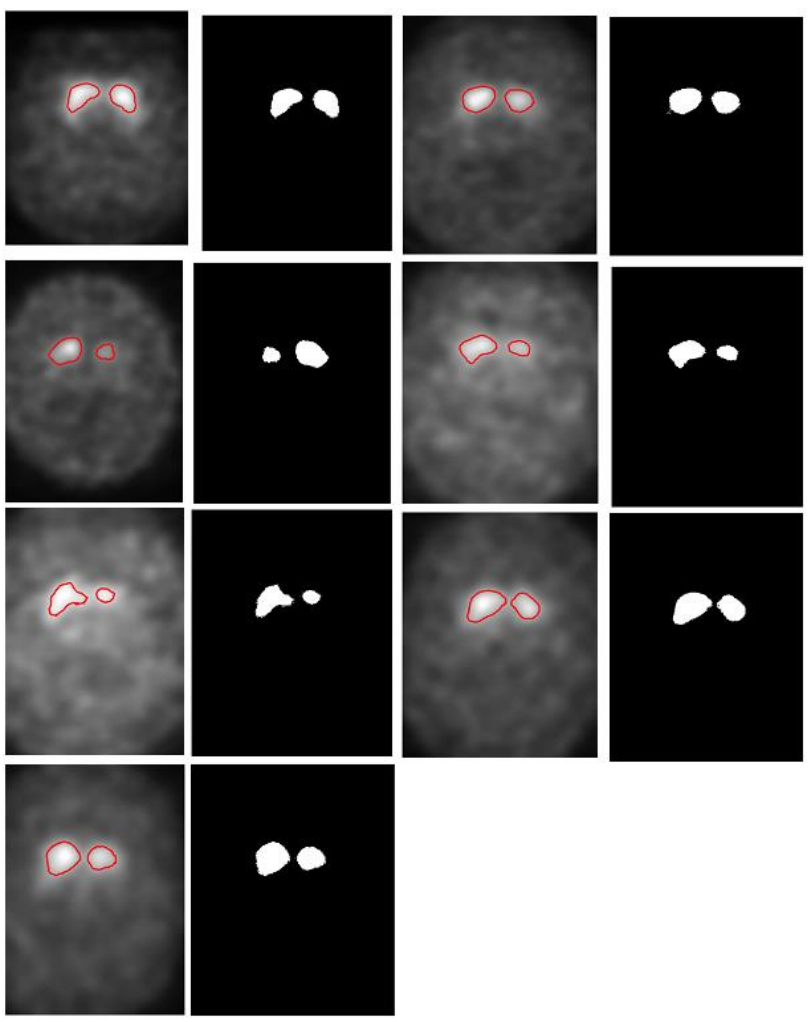

Figure 14: The boundary of ROI detected with corresponding binarized images

\subsection{Artificial Neural Network}

With the obtained data that is the area of Putamen and Caudate of left and right hemisphere of the brain we train a prediction model using Artificial Neural Network. We use supervised training method, where the classification of training data is included in the dataset. 


\subsubsection{Data for prediction model}

The earliest form of artificial neuron, the "Perceptron" proposed by Rosenblatt F. was in actual and modern sense an algorithm for learning binary classification. This binary classifier can be represented as a function that maps input $\mathrm{x}$ (real-valued) vector to an output $f(x)$. The function can be represented as following [24]

$$
f(x)= \begin{cases}1 & \text { if } w \times x+b>0 \\ 0 & \text { otherwise }\end{cases}
$$

Where $\mathrm{w}$ is a vector of weights, $w \times x$ is dot product $\sum(w i \times x i)$, and $b$ is the bias. The bias $b$ is like the constant $c$ at the end of the linear equation $y=a x+c$ for convenience of shaping the result for better representation of output. Doctors in real world diagnose Parkinson's disease on the basis of the degeneration of Putamen and Caudate nucleuses of the dorsal striatum. So the indicative feature of Parkinson's disease is the decrease in area of those regions. Thus our data for the neural network is the area of Putamen and Caudate of left and right hemispheres of brain that we find after processing of the SPECT images. We used that area data of left and right hemispheres of the brain as our main data for the prediction model.

For simplifying data more we scaled the data. We cropped the images to $720 \times 860$ pixels, and the area we got in pixels ranged from 1000-20000 pixels. For easier representation and faster calculation we scaled the data, so that we get the area in pixels ranging from 0-10 units. 1 unit is $=2000$ pixels. So the scaling factor " $s$ " of our data is as following:

$$
S=\frac{\text { area in pixels }}{2000}
$$

\subsubsection{Choosing activation function}

In our worked model, we used neural network model which can be simply represented as $f: x \rightarrow z$. Where,

$$
z=\sum(w \times i n p u t)+b
$$

Here "w" = weight of the node, and "b" is the bias [24]. The value of $Y$ can be from $-\infty$ to $+\infty$. which we bound within $0<\mathrm{w}<1$. For this bounding purpose many functions are used, and one of them is step function which can be represented as [24] 


$$
f(x)= \begin{cases}0 & \text { if } w \times x+b \leq 0 \\ 1 & \text { if } w \times x+b>0\end{cases}
$$

In case of using a step function our classification would have been a completely linear one. As our data isn't completely linear, as an activation function we used the sigmoid function. This makes the neural network we used a simple feed forward neural network or a sigmoid perceptron. A sigmoid function is defined as [25]

$$
\operatorname{sig}(z)=\frac{1}{1+e^{-z}}
$$

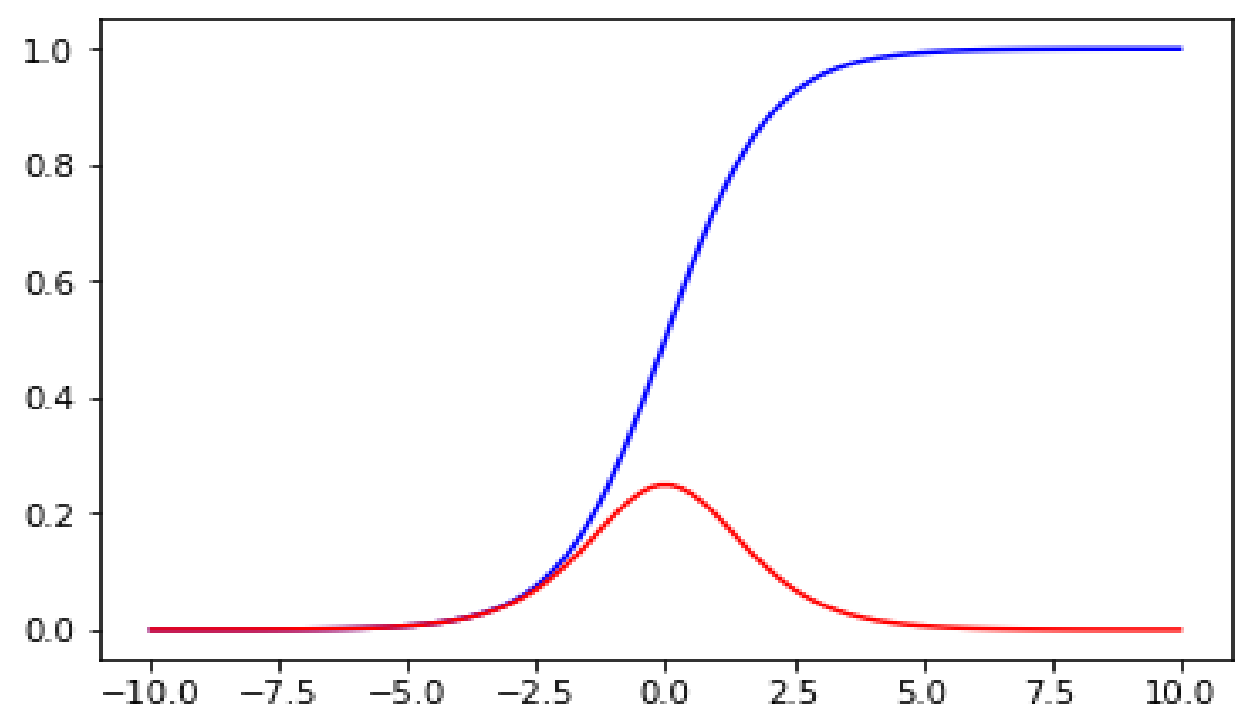

Figure 15: Sigmoid curve and Differential of Sigmoid Curve

We can see from the above sigmoid curve, and the function we used to represent our prediction model, $\mathrm{z}$ can be any number of the domain $\mathrm{R}$, and the number is large when there is more chance of Parkinson's disease and small when chance is less, and sigmoid (z) returns number close to 1 for large number, and a number close to 0 for smaller numbers. That's why sigmoid function is suitable for our method of classifying Parkinson's and not Parkinson's.

Another reason for using sigmoid is it's easy to find its derivative which is necessary for our learning function. Derivative of sigmoid can be defined as [25]

$$
\frac{d}{d x} \operatorname{sig}(x)=\operatorname{sig}(\mathrm{x})(1-\operatorname{sig}(\mathrm{x}))
$$


Sigmoid prepares the nonlinear model of classification and detection of PD.

\subsubsection{Cost function and training}

This ANN was trained on a known dataset with classification included in the dataset, in a form of supervised training. Here training of learning is the determination of weights for each processing elements, and optimizing the weight based on the cost function. The cost function determines the cost based on the difference of result found and desired result. We used the error squared function to find the Cost and weight needed for our network to work correctly. The cost function we used can be defined as [25]

$$
c(w, b) \equiv \sum(y(x)-t)^{2}
$$
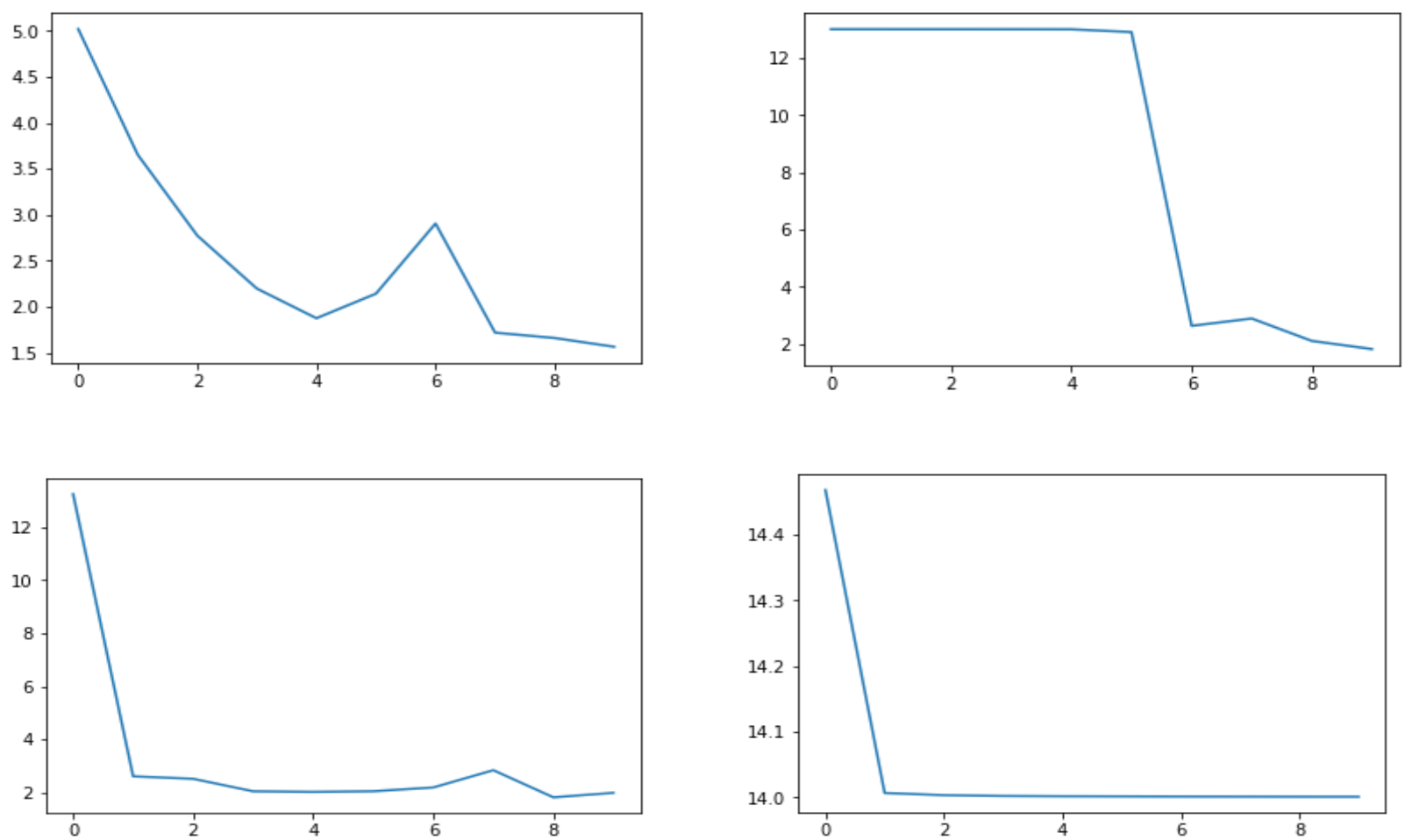

Figure 16: Changes in cost function for individual training

From the above equation of cost function we determine cost of individual neurons and reaching our guided output. By iterations for every input we reduce the cost function. It can be seen from equation 15, the lesser the cost function is the closer we are to our desired output. Below is the prediction model with the result positioned in predicted area. 

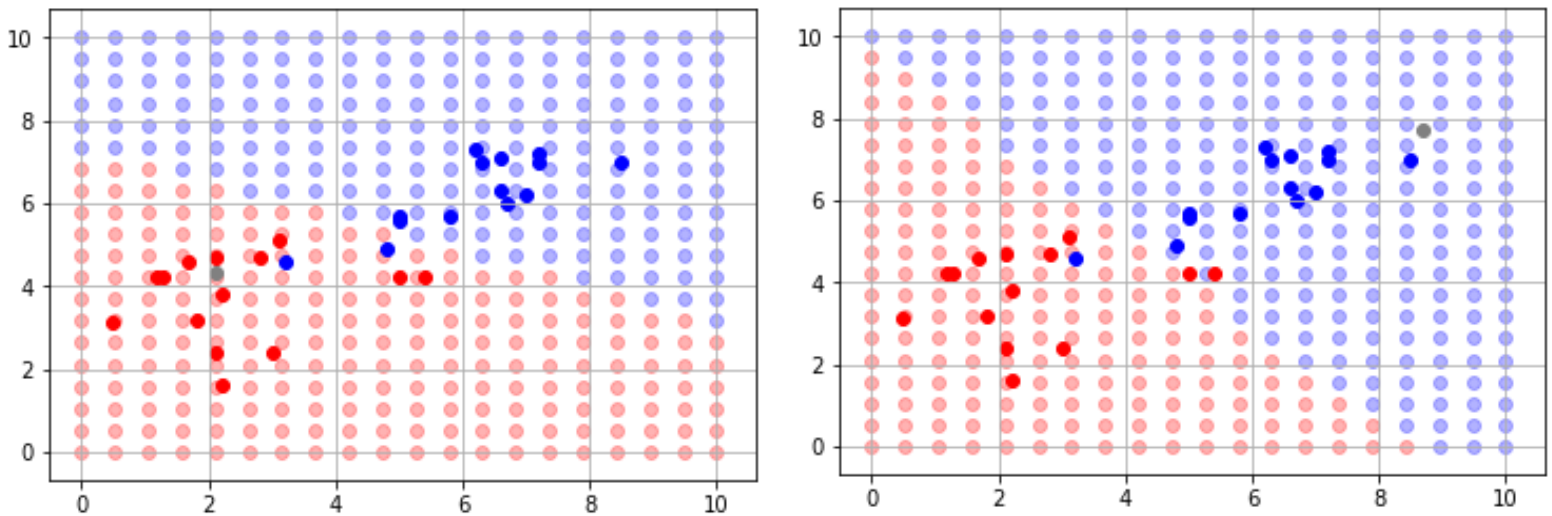

Figure 17 Results with test subject (in gray) in predicted area; red region is PD region and blue region is non-PD region formed my ANN 


\section{Chapter 4}

\section{Results}

\subsection{Image processing Result}

We obtained 200 SPECT images of subjects for training and evaluation. 150 of the 200 image were used for training the prediction model and the rest 50 were used to test the accuracy. Below are some sample images that detects the Putamen and Caudate regions of brain and calculate the bordered area (fig 18 and 19).
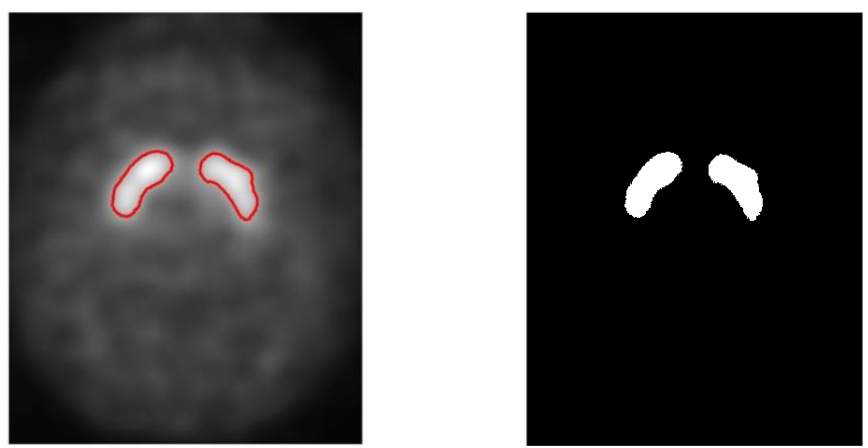

Figure 18 Area of Putamen and Caudate detection of non PD patient
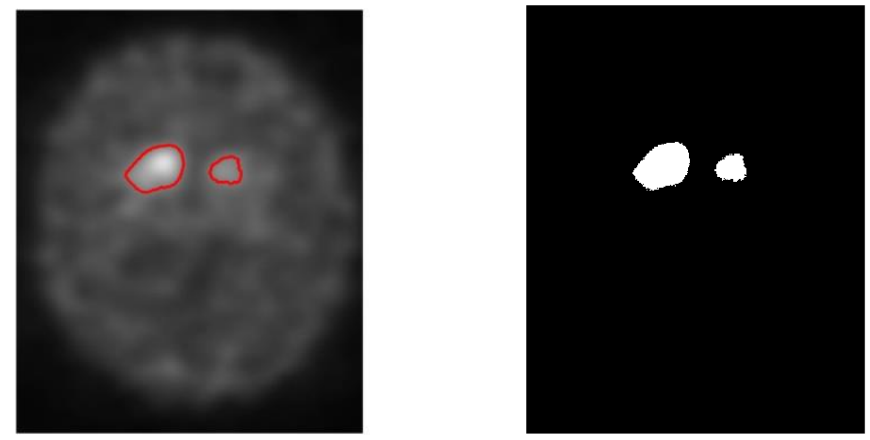

Figure 19: Area of Putamen and Caudate detection of patient with Parkinson's disease

For more accurate detection of the regions we used variable thresholds for converting the images into binary. The area of the brain regions for patient with Parkinson's disease varied from $2000-$ 9000 pixels, combined left and right hemisphere. For left hemisphere area ranged from 2500 - 
9000 with very few out of the range. For right hemisphere area ranged from 2000-7500. Below is the histogram comparing left and right hemisphere area of putamen and caudate of patient's with Parkinson's disease (fig 20).

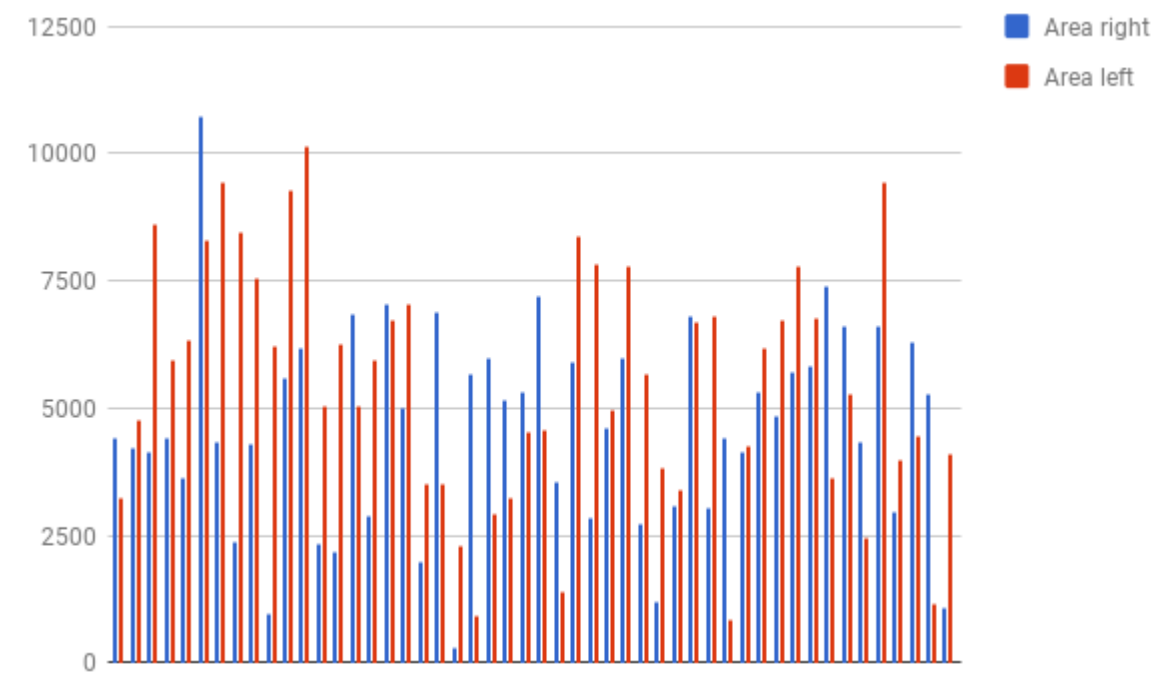

Figure 20: Left and Right area comparison of patients with Parkinson's

In case of control subjects that is subjects without Parkinson's disease, the left and right hemisphere area of Putamen and Caudate ranged from 8000-17000 pixels. Separately the left hemisphere size ranged from 7000-13000 with very few exceptions and the right hemisphere region size ranged from 8000-17000 with few exceptions. The slice 40 was used in more than $90 \%$ of the test images as slice 40 showed the region with most brightness and clarity. Below the histogram is comparison between left and right area of Putamen and Caudate of patients without Parkinson's disease (fig 21). 


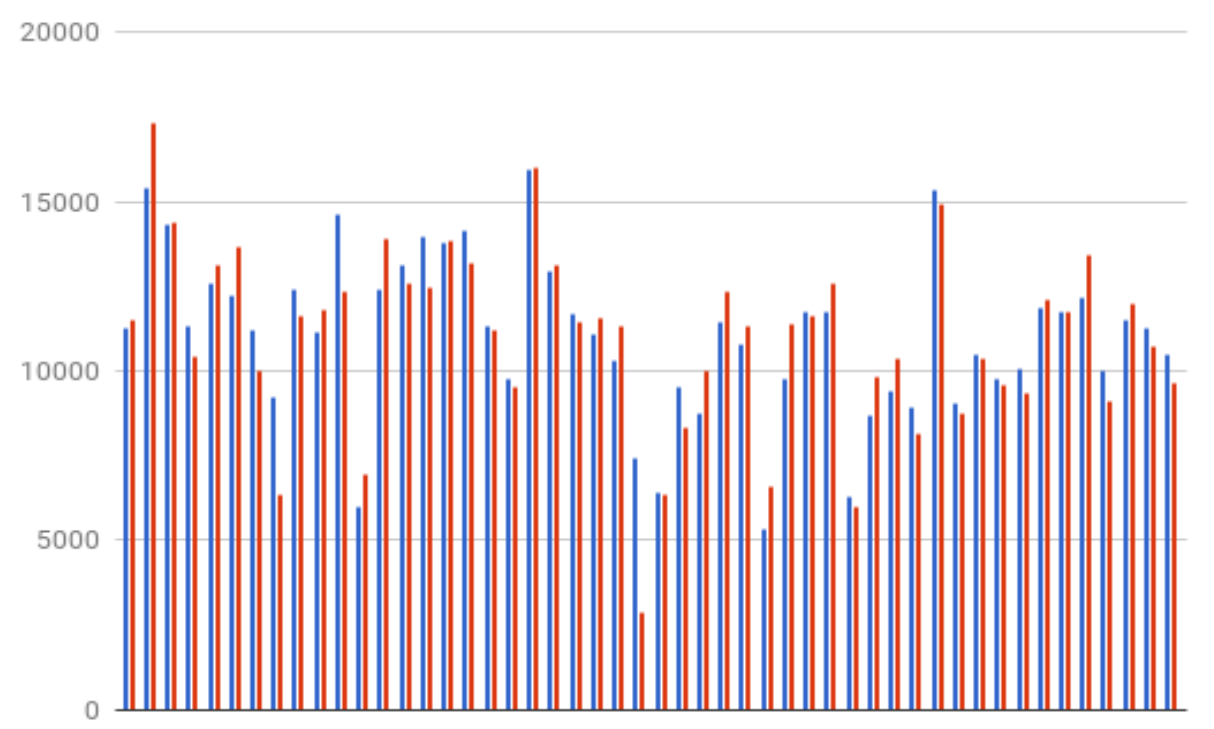

Figure 21: Left and Right area comparison of patients without Parkinson's disease

In the tables below we represent clinical data of some of the patients with both with Parkinson's disease and without Parkinson's disease. The time of these SPECT images taken ranged from 48 months to 12 months since the process of degeneration started.

\subsubsection{Tabular Representation of area of ROI in PD subjects}

\begin{tabular}{|c|c|c|c|c|c|c|c|c|c|}
\hline Patient Id & PD & Period & Area right & left & Threshold & Slice used & Time & Sex & Age \\
\hline 4111 & $\mathrm{P}$ & $29 / 01 / 2013$ & 4435 & 3250 & 0.45 & 40 & Screening & $\mathrm{M}$ & 74 \\
\hline 4111 & $\mathrm{P}$ & $02 / 06 / 2014$ & 4250 & 4783 & 0.5 & 40 & 12 Months & $\mathrm{M}$ & 75 \\
\hline 4112 & $\mathrm{P}$ & $15 / 02 / 2013$ & 4140 & 8632 & 0.65 & 40 & Screening & $\mathrm{M}$ & 54 \\
\hline 4112 & $\mathrm{P}$ & $18 / 03 / 2014$ & 4411 & 5943 & 0.68 & 40 & 12 Months & $\mathrm{M}$ & 55 \\
\hline 4112 & $\mathrm{P}$ & $27 / 03 / 2015$ & 3640 & 6345 & 0.65 & 40 & 24 Months & $\mathrm{M}$ & 56 \\
\hline 4122 & $\mathrm{P}$ & $02 / 06 / 2013$ & 10735 & 8326 & 0.56 & 40 & Screening & $\mathrm{M}$ & 64 \\
\hline
\end{tabular}




\begin{tabular}{|c|c|c|c|c|c|c|c|c|c|}
\hline Patient Id & $\begin{array}{c}\text { PD/Non } \\
\text { PD }\end{array}$ & Period & Area right & $\begin{array}{c}\text { Area } \\
\text { left }\end{array}$ & Threshold & Slice used & Time & Sex & Age \\
\hline 4122 & $\mathrm{P}$ & $03 / 19 / 2014$ & 4363 & 9446 & 0.66 & 40 & 36 Months & M & 65 \\
\hline 4122 & $\mathrm{P}$ & $04 / 08 / 2015$ & 2375 & 8450 & 0.78 & 40 & 48 Months & M & 66 \\
\hline 4123 & $\mathrm{P}$ & $22 / 01 / 2013$ & 4309 & 7575 & 0.7 & 40 & Screening & $\mathrm{F}$ & 60 \\
\hline
\end{tabular}

Table 1: Data table of patient's with Parkinson's disease

\subsubsection{Tabular Representation of area or ROI in Non-PD subjects}

\begin{tabular}{|l|l|l|l|l|l|}
\hline Patient Id & PD / Non PD & Area right & Area left & Threshold used & slice used \\
\hline 3318 & N & 11539 & 11311 & 0.53 & 40 \\
\hline 3222 & N & 17362 & 15455 & 0.5 & 40 \\
\hline 3318 & N & 14398 & 14349 & 0.48 & 40 \\
\hline 3464 & N & 10442 & 11378 & 0.59 & 40 \\
\hline 3466 & N & 13168 & 12638 & 0.55 & 40 \\
\hline 3055 & N & 13687 & 12225 & 0.57 & 40 \\
\hline 3301 & N & 6396 & 9250 & 0.66 & 40 \\
\hline 3310 & N & 11633 & 12451 & 0.58 & 40 \\
\hline 3428 & N & 11841 & 11199 & 0.52 & 40 \\
\hline 3457 & N & 12398 & 14676 & 0.58 & 40 \\
\hline 3478 & N & 6972 & 6000 & 0.68 & 40 \\
\hline
\end{tabular}




\begin{tabular}{|l|l|l|l|l|l|}
\hline Patient Id & PD / Non PD & Area right & Area left & Threshold used & slice used \\
\hline 3517 & $\mathrm{~N}$ & 13912 & 12433 & 0.53 & 40 \\
\hline 3523 & $\mathrm{~N}$ & 12595 & 13170 & 0.5 & 40 \\
\hline 3668 & $\mathrm{~N}$ & 12506 & 13991 & 0.55 & 40 \\
\hline
\end{tabular}

Table2: Data table of patient's without Parkinson's disease

\subsubsection{Comparison between Control and PD}

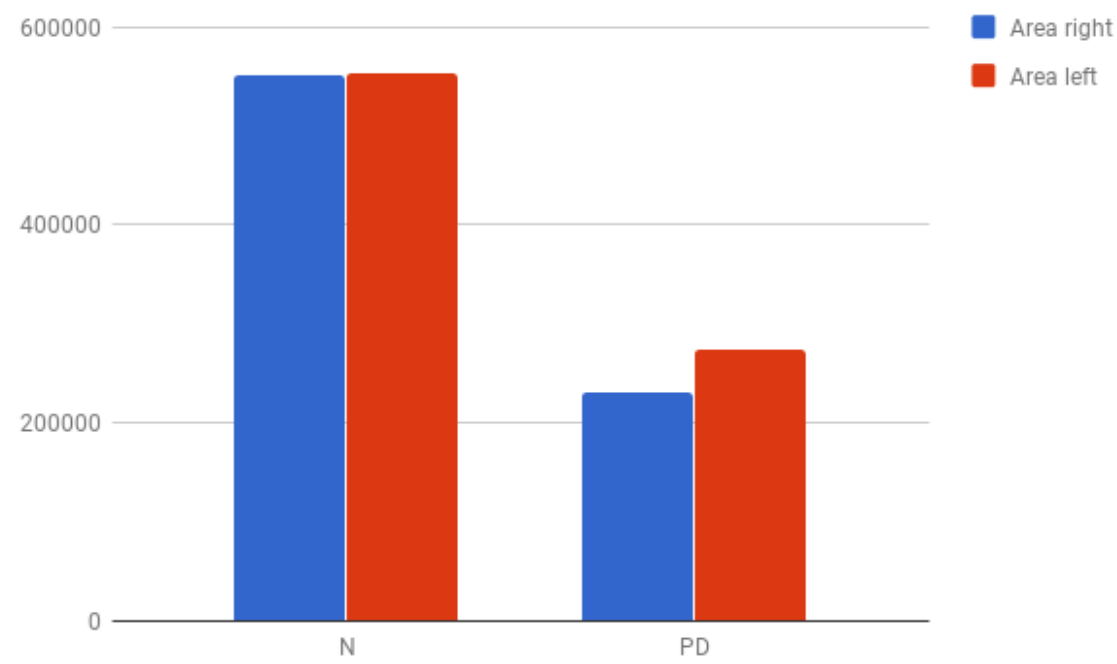

Figure 22: Comparison between sum of area right and left of the selected regions

Above is the histogram of comparison between the sums of area (fig 22). Below we represent comparison between area of left and right selected region of patient in tabular form.

\begin{tabular}{|c|c|c|c|c|c|c|c|}
\hline Patient Id & PD / N-PD & Area right & Area left & Patient Id & PD / N-PD & Area right & Area left \\
\hline 3318 & $\mathrm{~N}$ & 11539 & 11311 & 3208 & $\mathrm{~N}$ & 10030 & 11204 \\
\hline 4111 & $\mathrm{P}$ & 4435 & 3250 & 4123 & $\mathrm{~N}$ & 6396 & 9250 \\
\hline 3318 & $\mathrm{~N}$ & 14398 & 14349 & 3310 & $\mathrm{~N}$ & 11633 & 12451 \\
\hline
\end{tabular}




\begin{tabular}{|c|c|c|c|c|c|c|c|}
\hline Patient Id & PD / N-PD & Area right & Area left & Patient Id & PD / N-PD & Area right & Area left \\
\hline 3464 & $\mathrm{~N}$ & 10442 & 11378 & 3428 & $\mathrm{~N}$ & 11841 & 11199 \\
\hline 3466 & $\mathrm{~N}$ & 13168 & 12638 & 3457 & $\mathrm{~N}$ & 12398 & 14676 \\
\hline 3055 & $\mathrm{~N}$ & 13687 & 12225 & 3478 & $\mathrm{~N}$ & 6972 & 6000 \\
\hline 3517 & $\mathrm{~N}$ & 13912 & 12433 & 4112 & $\mathrm{P}$ & 4140 & 8632 \\
\hline 3523 & $\mathrm{~N}$ & 12595 & 13170 & 4112 & $\mathrm{P}$ & 4411 & 5943 \\
\hline 3668 & $\mathrm{~N}$ & 12506 & 13991 & 4112 & $\mathrm{P}$ & 3640 & 6345 \\
\hline 3950 & $\mathrm{~N}$ & 13841 & 13832 & 4122 & $\mathrm{P}$ & 10735 & 8326 \\
\hline 4008 & $\mathrm{~N}$ & 13238 & 14157 & 4122 & $\mathrm{P}$ & 4363 & 9446 \\
\hline 4063 & $\mathrm{~N}$ & 11258 & 11333 & 4122 & $\mathrm{P}$ & 2375 & 8450 \\
\hline 4140 & $\mathrm{~N}$ & 9542 & 9803 & 4123 & $\mathrm{P}$ & 4309 & 7575 \\
\hline 4118 & $\mathrm{~N}$ & 16050 & 15967 & 4121 & $\mathrm{P}$ & 971 & 6239 \\
\hline 3222 & $\mathrm{~N}$ & 17362 & 15455 & 3301 & $\mathrm{P}$ & 5586 & 9300 \\
\hline 4111 & $\mathrm{P}$ & 4250 & 4783 & 4123 & $\mathrm{P}$ & 6205 & 10167 \\
\hline
\end{tabular}

Table3: Data of patients with PD and without PD

\subsection{Results of NN}

In the tables below we represent part of the data we collected using images for testing our Neural Network.

\begin{tabular}{|l|l|r|r|r|r|l|l|}
\hline Patient Id & PD / Non PD & Area right & Area left & scaled right & Scaled Left & Prediction Value(z) & NN decision \\
\hline 3222 & $\mathrm{~N}$ & 17362 & 15455 & 8.7 & 8.7 & 0.0001353043 & Correct \\
\hline 3464 & $\mathrm{~N}$ & 10442 & 11378 & 5.2 & 5.2 & 0.0077435594 & Correct \\
\hline
\end{tabular}




\begin{tabular}{|c|c|c|c|c|c|c|c|}
\hline Patient Id & PD / Non PD & Area right & Area left & scaled right & Scaled Left & Prediction Value $(\mathrm{z})$ & $\mathrm{NN}$ decision \\
\hline 3055 & $\mathrm{~N}$ & 13687 & 12225 & 6.8 & 6.8 & 0.0004054995 & Correct \\
\hline 3310 & $\mathrm{~N}$ & 11633 & 12451 & 5.8 & 5.8 & 0.3872803995 & Correct \\
\hline 3428 & $\mathrm{~N}$ & 11841 & 11199 & 5.9 & 5.9 & 0.0075791362 & Correct \\
\hline 3478 & $\mathrm{~N}$ & 6972 & 6000 & 3.5 & 3.5 & 0.6962575018 & Correct \\
\hline 3523 & $\mathrm{~N}$ & 12595 & 13170 & 6.3 & 6.3 & 0.0590741376 & Correct \\
\hline 3950 & $\mathrm{~N}$ & 13841 & 13832 & 6.9 & 6.9 & 0.0018635586 & Correct \\
\hline 4063 & $\mathrm{~N}$ & 11258 & 11333 & 5.6 & 5.6 & 0.1487718734 & Correct \\
\hline 4118 & $\mathrm{~N}$ & 16050 & 15967 & 8.0 & 8.0 & 0.0005908602 & Correct \\
\hline 3013 & $\mathrm{~N}$ & 13173 & 13000 & 6.6 & 6.6 & 0.0628868045 & Correct \\
\hline 3072 & $\mathrm{~N}$ & 11570 & 11133 & 5.8 & 5.8 & 0.0060231601 & Correct \\
\hline 3173 & $\mathrm{~N}$ & 2903 & 7454 & 1.5 & 1.5 & 0.9716936493 & Wrong \\
\hline 3215 & $\mathrm{~N}$ & 8354 & 9533 & 4.2 & 4.2 & 0.2279100471 & Correct \\
\hline 3276 & $\mathrm{~N}$ & 12355 & 11473 & 6.2 & 6.2 & 0.0280599417 & Correct \\
\hline 3362 & $\mathrm{~N}$ & 6634 & 5377 & 3.3 & 3.3 & 0.8728029891 & Wrong \\
\hline 3424 & $\mathrm{~N}$ & 11658 & 11742 & 5.8 & 5.8 & 0.0714366800 & Correct \\
\hline 3453 & $\mathrm{~N}$ & 6020 & 6327 & 3.0 & 3.0 & 0.8860533626 & Wrong \\
\hline 3525 & $\mathrm{~N}$ & 10380 & 9411 & 5.2 & 5.2 & 0.0650774698 & Correct \\
\hline 3554 & $\mathrm{~N}$ & 14959 & 15369 & 7.5 & 7.5 & 0.0014421244 & Correct \\
\hline 3569 & $\mathrm{~N}$ & 10395 & 10481 & 5.2 & 5.2 & 0.0646219716 & Correct \\
\hline 3613 & $\mathrm{~N}$ & 9342 & 10090 & 4.7 & 4.7 & 0.4332663885 & Correct \\
\hline 3662 & $\mathrm{~N}$ & 11798 & 11765 & 5.9 & 5.9 & 0.0053969685 & Correct \\
\hline 3806 & $\mathrm{~N}$ & 9112 & 10027 & 4.6 & 4.6 & 0.1256528963 & Correct \\
\hline 3811 & $\mathrm{~N}$ & 10753 & 11271 & 5.4 & 5.4 & 0.0436162205 & Correct \\
\hline 4112 & PD & 4140 & 8632 & 2.1 & 4.3 & 0.9037121600 & Correct \\
\hline 4112 & PD & 4411 & 5943 & 2.2 & 3.0 & 0.9623863791 & Correct \\
\hline 4122 & PD & 4363 & 9446 & 2.2 & 4.7 & 0.8650011092 & Correct \\
\hline 3173 & PD & 2190 & 6255 & 1.1 & 3.1 & 0.9934593486 & Correct \\
\hline
\end{tabular}




\begin{tabular}{|c|c|c|c|c|c|c|c|}
\hline Patient Id & PD / Non PD & Area right & Area left & scaled right & Scaled Left & Prediction Value(z) & NN decision \\
\hline 3184 & PD & 2910 & 5974 & 1.5 & 3.0 & 0.9934593486 & Correct \\
\hline 3233 & PD & 5004 & 7038 & 2.5 & 3.5 & 0.9557180515 & Correct \\
\hline 3279 & PD & 2000 & 3525 & 1.0 & 1.8 & 0.9972797967 & Correct \\
\hline 3377 & PD & 6884 & 3530 & 3.4 & 1.8 & 0.8089593774 & Correct \\
\hline 3386 & PD & 309 & 2321 & 0.2 & 1.2 & 0.9988986584 & Correct \\
\hline 3445 & PD & 6009 & 2952 & 3.0 & 1.5 & 0.9970302819 & Correct \\
\hline 3567 & PD & 5329 & 4554 & 2.7 & 2.3 & 0.9964634089 & Correct \\
\hline 3622 & PD & 3582 & 1418 & 1.8 & 0.7 & 0.9914228180 & Correct \\
\hline 3634 & PD & 2862 & 7821 & 1.4 & 3.9 & 0.9833660778 & Correct \\
\hline 3762 & PD & 5995 & 7779 & 3.0 & 3.9 & 0.7926817834 & Correct \\
\hline 4020 & PD & 1213 & 3838 & 0.6 & 1.9 & 0.9954048319 & Correct \\
\hline 4056 & PD & 6835 & 6683 & 3.4 & 3.3 & 0.6150896230 & Correct \\
\hline 4062 & PD & 4431 & 879 & 2.2 & 0.4 & 0.9950345596 & Correct \\
\hline 4103 & PD & 5334 & 6172 & 2.7 & 3.1 & 0.9414615741 & Correct \\
\hline 4103 & PD & 5714 & 7796 & 2.9 & 3.9 & 0.7539213521 & Correct \\
\hline 3154 & PD & 7415 & 3629 & 3.7 & 1.8 & 0.8500292297 & Correct \\
\hline 3203 & PD & 4331 & 2465 & 2.2 & 1.2 & 0.9876857180 & Correct \\
\hline 3367 & PD & 2964 & 3980 & 1.5 & 2.0 & 0.9931141258 & Correct \\
\hline 3374 & PD & 6327 & 4474 & 3.2 & 2.2 & 0.8820899981 & Correct \\
\hline 3380 & PD & 5280 & 1189 & 2.6 & 0.6 & 0.9480580527 & Correct \\
\hline 3403 & PD & 1077 & 4100 & 0.5 & 2.1 & 0.9982600093 & Correct \\
\hline
\end{tabular}

Table4: ANN result in tabular form

Here is the prediction model with the area in red representing the subjects with Parkinson's disease and the area in blue representing the subjects without PD. The test PD is indicated in gray and put in the predicted area (fig 23 and 24). 

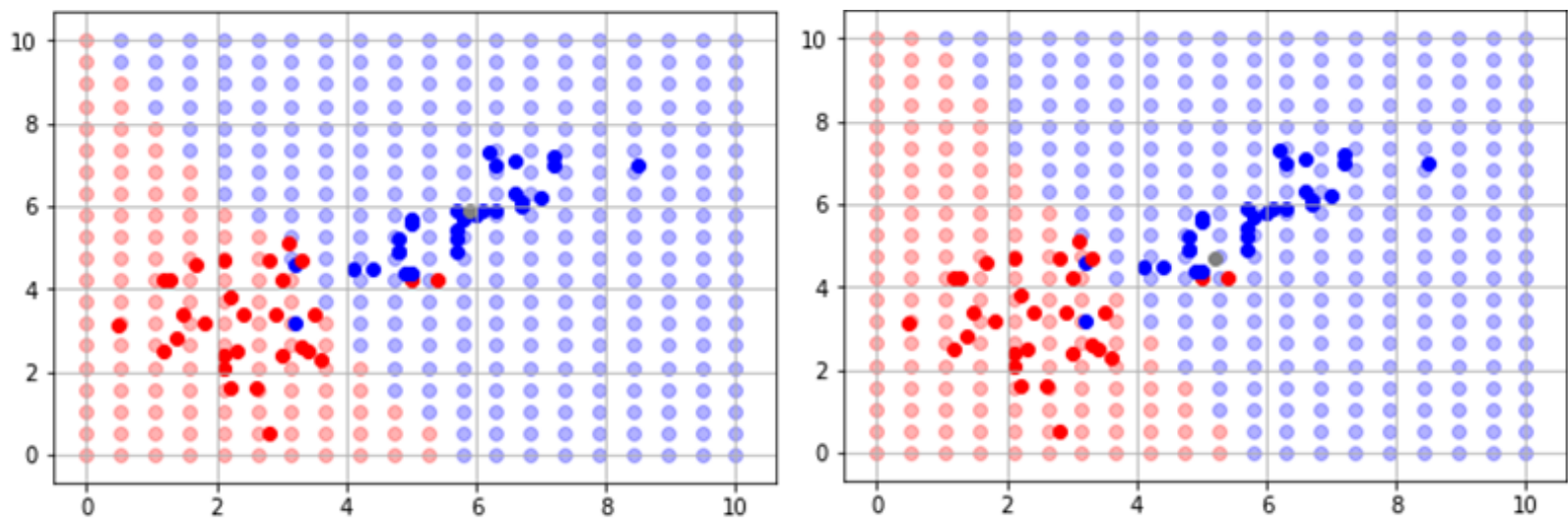

Figure 23: Results for non-PD subjects accurately predicted by ANN
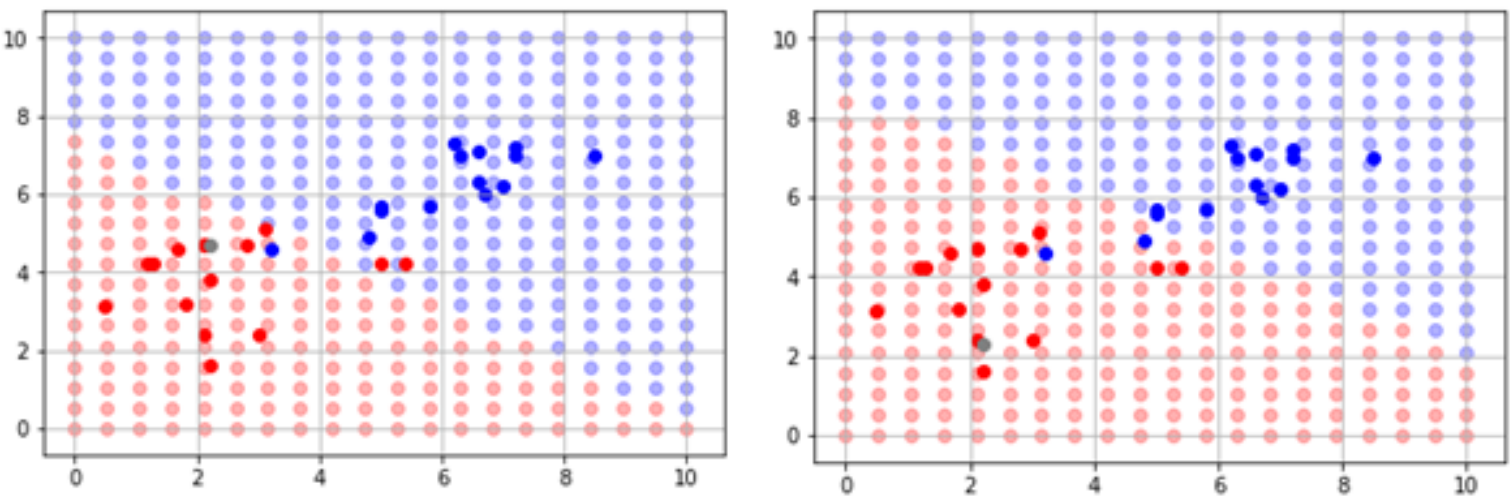

Figure 24 Results showing PD subjects accurately predicted by ANN

\subsection{Accuracy, sensitivity, Specificity}

Out of 50 test images of both PD and Non PD 3 results were wrong. All the PDs could successfully be identified. 3 Controls were wrongly identified as PD.

True Positive (TP): Number of cases correctly identified as PD

False Positive (FP): Number of cases incorrectly identified as PD

True Negative (TN): Number of cases correctly identified as healthy

False Negative (FN): Number of cases incorrectly identified as healthy. 


\begin{tabular}{|l|r|}
\hline True positive & 25 \\
\hline False positive & 3 \\
\hline True negative & 22 \\
\hline False negative & 0 \\
\hline
\end{tabular}

Table5: Classified Result

$$
\begin{aligned}
& \text { Accuracy } \%=\frac{T P+T N}{T P+T N+F P+F N} * 100 \\
& \text { Sensitivity } \%=\frac{T P}{T P+F N} * 100 \\
& \text { Specificity } \%=\frac{T N}{T N+F P} * 100
\end{aligned}
$$

According to the equation 16, 17 and 18 [26] accuracy was found to be 94\%, Sensitivity was found to be $100 \%$ and Specificity was found to be $88 \%$.

\section{Here we represent the pie chart of accuracy:}

\section{Count of NN decision}

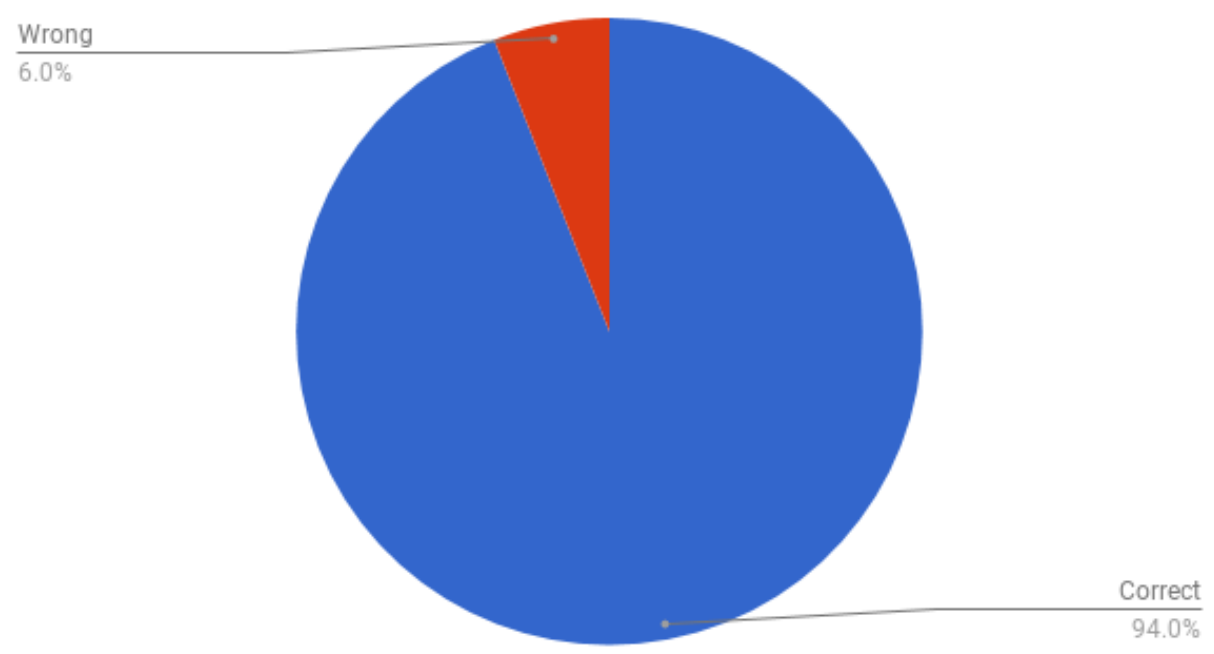

Figure 25: Accuracy of result 


\section{Chapter 5}

\section{Conclusion}

Early diagnosis of Parkinson's is most important for the patient to reduce its impact. We presented in this paper a prediction model with the aid of artificial intelligence to improve over human accuracy and provide with the scope of early detection. With our proposed prediction model we aim to make it easier for doctors to do precise diagnosis and prediction of $\mathrm{PD}$, both of which have human limitations due to the method of detection of PD that is used now. We separated the work of detection and prediction methods to detect and measure the area of brain that is affected due to PD, and use that data in a neural network to create the prediction model. In this proposed method accuracy of $94 \%$, sensitivity of $100 \%$, and specificity of $88 \%$ were achieved. The contributions of this study are as following (i)This model is highly efficient to aid doctors as it uses only 2 features. (ii)The sample size used was large and very accurate, which makes this model very accurate. (iii)Using image processing and neural network separately makes application of this model easier in real world situations, i.e., this approach takes very less processing power and its accuracy is reliable. It can be inferred from this proposed model that, area analysis and use of simple neural network is useful in developing prediction models that can help a doctor reduce the long process of diagnosis and eradicate any human error.

\subsection{Future work}

The model presented in this paper looked at the problem of detecting Parkinson's disease from a different angle than existing approaches, but the results obtained are 
very much applicable to real life situations. These results are not the means to an end, rather many works lay ahead to build up this model to be actually implemented in real life situation. A model with more dataset can give an even more reliable accuracy. Also, comparing this model with other existing approaches on the premises of accuracy, efficiency and applicability on real life situations can better reveal the position of this approach. Building up a usable software with this approach can readily assist physicians to detect Parkinson's with better accuracy than before. More works can be done on datasets with wider variety of subjects, and also adding more factors that come to play in regards of the disease. A big factor in this disease is the lack awareness, and also that complication arises or be noticeable only after a certain amount of time has passed, and degeneration of neurons have already started in the subject. So datasets with images taken earlier than the one we used, if can be obtained, can give an even better outcome. 


\section{Reference}

1. K. Mamun, "Deep brain stimulation: a light of hope for Parkinson's patients", Deep brain stimulation: a light of hope for Parkinson's patients | theindependentbd.com, 2018. [Online]. Available: http://www.theindependentbd.com/arcprint/details/115731/2017-0925.

2. K. Jellinger, G. Logroscino, G. Rizzo, M. Copetti, S. Arcuti, D. Martino and A. Fontana, "Accuracy of clinical diagnosis of Parkinson disease: A systematic review and metaanalysisAuthor Response", Neurology, vol. 87, no. 2, pp. 237-238, 2016

3. K. N. R. Challa, V.S. Pagolu, G. Panda, An Improved Approach for Prediction of Parkinson's Disease using Machine Learning Techniques, 2016.

4. H. Choi, S. Ha, H. Im, S. Paek and D. Lee, "Refining diagnosis of Parkinson's disease with deep learning-based interpretation of dopamine transporter imaging", NeuroImage: Clinical, vol. 16, pp. 586-594, 2017.

5. A. Rahmim, P. Huang, N. Shenkov, S. Fotouhi, E. Davoodi-Bojd, L. Lu, Z. Mari, H. Soltanian-Zadeh and V. Sossi, "Improved prediction of outcome in Parkinson's disease using radiomics analysis of longitudinal DAT SPECT images", NeuroImage: Clinical, vol. 16, pp. 539-544, 2017.

6. P. Acton and A. Newberg, "Artificial neural network classifier for the diagnosis of Parkinson's disease using [99mTc]TRODAT-1 and SPECT", Physics in Medicine and Biology, vol. 51, no. 12, pp. 3057-3066, 2006.

7. R. Prashanth, S.D. Roy, P. K. Mandal, S. Ghosh, Automatic classification and prediction models for early Parkinson's disease diagnosis from SPECT imaging.Expert systems with Applications 41 (3333-3342), 2014.

8. F. Oliveira, D. Faria, D. Costa, M. Castelo-Branco and J. Tavares, "Extraction, selection and comparison of features for an effective automated computer-aided diagnosis of Parkinson's disease based on [123I]FP-CIT SPECT images", European Journal of Nuclear Medicine and Molecular Imaging, 2017.

9. I. Illán, J. Górriz, J. Ramírez, F. Segovia, J. Jiménez-Hoyuela and S. Ortega Lozano, "Automatic assistance to Parkinson's disease diagnosis in DaTSCAN SPECT imaging", Medical Physics, vol. 39, no. 10, pp. 5971-5980, 2012. 
10. T. Booth, M. Nathan, A. Waldman, A. Quigley, A. Schapira and J. Buscombe, "The Role of Functional Dopamine-Transporter SPECT Imaging in Parkinsonian Syndromes, Part 2", American Journal of Neuroradiology, vol. 36, no. 2, pp. 236-244, 2014.

11. "Grayscale Morphology." National Institutes of Health, U.S. Department of Health and Human Services, imagej.nih.gov/ij/plugins/gray-morphology.html.

12. Kundu, Abhijit, et al. "Feature Space Optimization for Semantic Video Segmentation." 2016 IEEE Conference on Computer Vision and Pattern Recognition (CVPR), 2016, doi:10.1109/cvpr.2016.345.22

13. 2018. [Online]. Available: https://mayo.edu/research/departments-divisions/departmentneurology/programs/movement-disorders.

14. U.Sinha, "Image Moments.” AI Shack, aishack.in/tutorials/image-moments/.

15. "K-Means Clustering in Python”, 2018, [Online].Available: https://mubaris.com/2017/10/01/kmeans-clustering-in-python/

16. "Parkinson's Progression Markers Initiative |", Ppmi-info.org, 2018. [Online]. Available: http://www.ppmi-info.org/

17. J. Bobin, J. L. Starck, J. M. Fadili, Y. Moudden and D. L. Donoho, "Morphological Component Analysis: An Adaptive Thresholding Strategy," in IEEE Transactions on Image Processing, vol. 16, no. 11, pp. 2675-2681, Nov. 2007.doi:

10.1109/TIP.2007.907073

18. A. Martelli, "An application of heuristic search methods to edge and contour detection", Communications of the ACM, vol. 19, no. 2, pp. 73-83, 1976.

19. F. Leymarie and M. Levine, "Simulating the grassfire transform using an active contour model", IEEE Transactions on Pattern Analysis and Machine Intelligence, vol. 14, no. 1, pp. 56-75, 1992.

20. Changjiang Zhang, Xiaodong Wang, Haoran Zhang, G. Lv and Han Wei, "A Reducing Multi-Noise Contrast Enhancement Algorithm for Infrared Image," First International Conference on Innovative Computing, Information and Control - Volume I (ICICIC'06), Beijing, 2006, pp. 632-635. doi: 10.1109/ICICIC.2006.23 
21. S. Satpathy, M. C. Pradhan and S. Sharma, "Comparative Study of Noise Removal Algorithms for Denoising Medical Image Using LabVIEW," 2015 International Conference on Computational Intelligence and Communication Networks (CICN), Jabalpur, 2015, pp. 300-305.doi: 10.1109/CICN.2015.67

22. D. G. Bailey, C. T. Johnston and Ni Ma, "Connected components analysis of streamed images," 2008 International Conference on Field Programmable Logic and Applications, Heidelberg, 2008, pp. 679-682. doi: 10.1109/FPL.2008.4630038

23. S. m. Kang, J. h. Kim, Z. Yuan, S. h. Song and J. d. Cho, "A fast region expansion labeling of connected components in binary image," The 18th IEEE International Symposium on Consumer Electronics (ISCE 2014), JeJu Island, 2014, pp. 1-2. doi: 10.1109/ISCE.2014.6884436

24. "Artificial neural network", En.wikipedia.org, 2018. [Online]. Available: https://en.wikipedia.org/wiki/Artificial_neural_network.

25. Nielsen, M. (2018). Neural Networks and Deep Learning. [online] Neuralnetworksanddeeplearning.com. Available at: http://neuralnetworksanddeeplearning.com/chap1.html

26. Alireza Baratloo, G. (2018). Part 1: Simple Definition and Calculation of Accuracy, Sensitivity and Specificity. [online] PubMed Central (PMC). Available at: https://www.ncbi.nlm.nih.gov/pmc/articles/PMC4614595 